\title{
RATIONAL SZEGÖ QUADRATURES ASSOCIATED WITH CHEBYSHEV WEIGHT FUNCTIONS
}

\author{
ADHEMAR BULTHEEL, RUYMÁN CRUZ-BARROSO, KARL DECKERS, \\ AND PABLO GONZÁLEZ-VERA
}

\begin{abstract}
In this paper we characterize rational Szegő quadrature formulas associated with Chebyshev weight functions, by giving explicit expressions for the corresponding para-orthogonal rational functions and weights in the quadratures. As an application, we give characterizations for Szegö quadrature formulas associated with rational modifications of Chebyshev weight functions. Some numerical experiments are finally presented.
\end{abstract}

\section{INTRODUCTION}

Szegó quadrature formulas approximate integrals over the complex unit circle $\mathbb{T}:=\{z \in \mathbb{C}:|z|=1\}$ and are the analogs of Gauss quadrature formulas that approximate integrals over an interval $I:=[-1,1]$. That is,

$$
\int f(z) d \mu(z) \approx \sum_{j=1}^{n} \lambda_{j} f\left(z_{j}\right)
$$

where $\mu$ is a positive measure on $\mathbb{T}$, respectively $I$.

Gauss quadrature formulas are optimal in the sense that nodes $z_{j} \in I$ and weights $\lambda_{j}>0$ are chosen such that the quadrature formula is exact for all functions $f \in \mathbb{P}_{2 n-1}$ that are polynomials of degree up to $2 n-1$ and it is not possible to construct a quadrature formula of this form that is exact for all polynomials of degree $2 n$. They have a maximal domain of validity. Therefore, the nodes have to be chosen as the zeros of the $n$th polynomial orthogonal with respect to the inner product $\langle f, g\rangle=\int f(z) g(z) d \mu(z)$. These zeros are all simple and in $I$.

On the unit circle, one needs to define a positive definite Hermitian inner product $\langle f, g\rangle=\int f(z) \overline{g(z)} d \mu(z)$. The $n$th orthogonal polynomial with respect to this inner product has all its zeros inside the open unit disk, and thus, they are not very useful as nodes of a quadrature formula. Here, the para-orthogonal polynomials are

Received by the editor May 5, 2008.

2000 Mathematics Subject Classification. Primary 42C05, 65D32.

Key words and phrases. Rational Szegö quadrature formulas, Szegö quadrature formulas, orthogonal rational functions, Chebyshev weight functions.

The work of the first three authors was partially supported by the Fund of Scientific Research (FWO), project "RAM: Rational modelling: optimal conditioning and stable algorithms", grant \#G.0423.05 and the Belgian Network DYSCO (Dynamical Systems, Control, and Optimization), funded by the Interuniversity Attraction Poles Programme, initiated by the Belgian State, Science Policy Office. The scientific responsibility rests with the authors.

The work of the last author was partially supported by the research project MTM 2005-08571 of the Spanish Government.

(C)2008 American Mathematical Society Reverts to public domain 28 years from publication 
producing the nodes that are needed. Para-orthogonal polynomials are orthogonal to all polynomials of lower degree, except the constants. All their zeros are simple and on $\mathbb{T}$. These para-orthogonal polynomials have one free parameter $\tau \in \mathbb{T}$. When using the zeros of the $n$th para-orthogonal polynomials, then there exist positive weights such that the quadrature formula is exact for all $f \in \mathcal{R}_{n-1}$, that is, the space of all Laurent polynomials of degree at most $n-1$, again a space of dimension $2 n-1$. Like for Gaussian formulas, this space is a maximal domain of validity since it is not possible to have an $n$-point quadrature formula with distinct nodes and positive weights that is exact in a space of Laurent polynomials of a larger degree neither for the positive or the negative powers of $z$. The Szegő quadrature formulas were first studied by Jones, Njåstad, and Thron 25] in connection with the trigonometric moment problem.

The Joukowski transform is a map between the unit circle and the interval $[-1,1]$. It implies a relationship between the Szegö and the Gauss quadrature formulas. In fact, this was already studied by Szegö [28, Section 11.5] and Geronimus [21, Chapter 9]. For $\tau=1$, a $2 n$-point Szegö formula results in an $n$-point Gauss quadrature formula and for $\tau=-1$, a $(2 n+2)$-point Szegö formula can be related to an $n$-point Gauss-Lobatto formula, having two extra nodes in the endpoints of the interval. Also Gauss-Radau formulas can be obtained taking $(2 n+1)$-point Szegö formulas and $\tau= \pm 1$, which fixes one of the endpoints of the interval; see [1. For other values of $\tau$, the quadrature on the interval is not optimal; see [2]. Szegö-Lobatto and Szegö-Radau formulas on the unit circle were recently discussed by Jagels and Reichel [24].

This theory has been generalized in a sequence of papers by Bultheel et al. to the case where the (orthogonal) polynomials in the previous theory are replaced by (orthogonal) rational functions having prescribed poles outside the closed unit disk. If all these poles are at infinity, the polynomials reappear as a special case. For a comprehensive survey see $[5$. See also $[6$ for a survey.

So the Szegö quadrature formulas are replaced by rational Szegő quadrature formulas. If $\mathcal{L}_{n}$ is the space of rational functions of degree $n$ at most whose poles are at $\left\{1 / \bar{\alpha}_{k}: k=1, \ldots, n\right\}$ and $\mathcal{L}_{n *}$ is the space of rational functions of degree $n$ at most whose poles are at $\left\{\alpha_{k}: k=1, \ldots, n\right\}$, then the rational Szegő quadrature formulas are exact in the space $\mathcal{R}_{n-1}=\mathcal{L}_{n-1}+\mathcal{L}_{(n-1) *}$. The nodes are the zeros of the $n$th rational para-orthogonal function that depends, as in the polynomial case, on a parameter $\tau \in \mathbb{T}$.

A relation between orthogonal rational functions on the interval and on the unit circle has been discussed in [16] and [32]. Rational Szegö-Lobatto and Szegö-Radau formulas have been recently studied in [7.

Gauss-Chebyshev quadrature formulas are associated with a measure that is generated by one of the Chebyshev weights. These weights are among the rare examples where a translation to the unit circle gives explicit expressions for the orthogonal polynomials. This is also true for the orthogonal rational functions, which have been under investigation with respect to rational Gauss-Chebyshev quadrature; see e.g. [15], 17, [18, 20], 29] and [30. To get more general cases where explicit expressions are obtained, a technique of rational modifications of these Chebyshev weights are considered in [14].

In this paper we will continue in this line of developments by considering the construction of explicit expressions for the para-orthogonal rational functions and 
for the weights in rational Szegö quadrature formulas that result from Chebyshev weights as they are translated into the unit circle. As an application, characterization results for Szegö quadrature formulas associated with rational modifications of these Chebyshev weights are obtained.

The outline of this paper is as follows. After giving the theoretical preliminaries in Section 2, in Section 3 we give explicit expressions for the orthogonal rational functions on the unit circle, associated with Chebyshev weight functions. Next, in Sections 4 and 5 we characterize rational Szegő quadrature formulas associated with Chebyshev weight functions, respectively, Szegő quadrature formulas associated with rational modifications of Chebyshev weight functions. We conclude with some numerical examples.

\section{Preliminaries}

Suppose $\mu$ is a positive bounded Borel measure on $[-\pi, \pi]$, and consider the general framework of the approximation of integrals on the unit circle $\mathbb{T}$ in the complex plane, i.e., integrals of the form 1

$$
I_{\mu}(f):=\int_{-\pi}^{\pi} f\left(e^{\mathbf{i} \theta}\right) d \mu(\theta)=\int_{\mathbb{T}} f(z) d \mu(z) .
$$

As usual, estimations of $I_{\mu}(f)$ are produced when replacing $f(z)$ in (11) by an appropriate approximating (interpolating) function $L(z)$, so that $I_{\mu}(L)$ can now be easily computed. Let $\Lambda:=\mathbb{C}\left[z, z^{-1}\right]$ denote the complex vector space of Laurent polynomials in the variable $z$. We then set $\Lambda_{p, q}:=\operatorname{span}\left\{z^{p}, \ldots, z^{q}\right\}$ for $p, q \in \mathbb{Z}$, with $p \leq q$. Because of the density of $\Lambda$ in $C(\mathbb{T})=\{f: \mathbb{T} \rightarrow \mathbb{C}, f$ is continuous $\}$ with respect to the uniform norm (see e.g. [13, pp. 304-305]), it seems reasonable to approximate $f(z)$ in (11) by an appropriate Laurent polynomial. This way, the so-called "quadrature formulas on the unit circle", or "Szego" rules", introduced in [25] (see also [22, [23, Chapter 4] and 27]) and of the form

$$
I_{n}(f):=\sum_{j=1}^{n} \lambda_{j} f\left(z_{j}\right), \quad z_{j} \in \mathbb{T}, \quad j=1, \ldots, n, \quad z_{j} \neq z_{k} \text { if } j \neq k,
$$

appear as the analogue on $\mathbb{T}$ of the Gaussian formulas when dealing with the estimation of integrals with respect to a measure supported on an interval $[a, b]$, with $-\infty \leq a<b \leq+\infty$ (see e.g. [19]).

Consider now the Hilbert space $L_{2}^{\mu}(\mathbb{T})$ of measurable functions $\phi$ for which $\int_{-\pi}^{\pi}\left|\phi\left(e^{\mathbf{i} \theta}\right)\right|^{2} d \mu(\theta)<+\infty$. Then the inner product induced by $\mu$ is given by

$$
\langle\phi, \psi\rangle_{\mu}=\int_{-\pi}^{\pi} \phi\left(e^{\mathbf{i} \theta}\right) \overline{\psi\left(e^{\mathbf{i} \theta}\right)} d \mu(\theta), \quad \phi, \psi \in L_{2}^{\mu}(\mathbb{T}) .
$$

In this paper we will deal with the more general framework of orthogonal rational functions (ORFs). Suppose a sequence of complex numbers $\mathcal{A}=\left\{\alpha_{1}, \alpha_{2}, \ldots\right\} \subset \mathbb{D}$ is given, and define the Blaschke factors

$$
\zeta_{k}(z)=\frac{z-\alpha_{k}}{1-\overline{\alpha_{k}} z}, \quad k=1,2, \ldots,
$$

and Blaschke products

$$
B_{0}(z) \equiv 1, \quad B_{k}(z)=B_{k-1}(z) \zeta_{k}(z), \quad k=1,2, \ldots
$$

\footnotetext{
${ }^{1}$ The measure $\mu$ on $[-\pi, \pi]$ induces a measure on $\mathbb{T}$ for which we shall use the same notation $\mu$.
} 
The space of rational functions with poles in $\left\{1 / \overline{\alpha_{1}}, \ldots, 1 / \overline{\alpha_{n}}\right\}$ is then defined as

$$
\mathcal{L}_{n}=\operatorname{span}\left\{B_{0}(z), \ldots, B_{n}(z)\right\},
$$

where we set $\mathcal{L}_{-1}=\emptyset$ to be the trivial subspace. This way, the ordinary polynomial situation is recovered by taking $\alpha_{k}=0$ for every $k=1,2, \ldots$, so that $\zeta_{k}(z)=z$ and $B_{k}(z)=z^{k}$.

We define the substar conjugate of a function $f$ as $f_{*}(z)=\overline{f(1 / \bar{z})}$, and the super-star conjugation of a function $f_{n} \in \mathcal{L}_{n}$ as

$$
f_{n}^{*}(z)=B_{n}(z) f_{n *}(z) .
$$

Note that $f_{*}(z)=\overline{f(z)}$ whenever $z \in \mathbb{T}$. Furthermore, for a given polynomial $P_{n}(z)=\sum_{k=0}^{n} c_{k} z^{k}$ of exact degree $n$, the super-star conjugate 2 is given by $P_{n}^{*}(z)=$ $\sum_{k=0}^{n} \overline{c_{n-k}} z^{k}$. Consequently, define

$$
\pi_{0}(z) \equiv 1, \quad \pi_{k}(z)=\prod_{j=1}^{k}\left(1-\overline{\alpha_{j}} z\right), \quad k=1,2, \ldots,
$$

and let $\mathbb{P}_{n}$ represent the space of polynomials of degree less than or equal to $n$. Then, we equivalently have for every $k=1,2, \ldots$ that

$$
B_{k}(z)=\frac{\pi_{k}^{*}(z)}{\pi_{k}(z)}
$$

and

$$
\mathcal{L}_{n}=\left\{\frac{p_{n}(z)}{\pi_{n}(z)}: p_{n} \in \mathbb{P}_{n}\right\} .
$$

For a fixed natural number $n$ we obtain a set of orthonormal rational functions $\left\{\chi_{k}(z)\right\}_{k=0}^{n}$ by orthonormalizing the basis $\left\{B_{k}(z)\right\}_{k=0}^{n}$ (in this order) with respect to the measure $\mu$ and inner product given by (3). Note that $\chi_{n}(z)=\sum_{k=0}^{n} a_{k} B_{k}(z)$ is uniquely determined if we assume the leading coefficient $a_{n}$ to be strictly positive. Repeating the process for every $n$, an orthonormal system $\left\{\chi_{k}(z)\right\}_{k=0}^{\infty}$ is obtained, so that $\chi_{n} \in \mathcal{L}_{n} \backslash \mathcal{L}_{n-1}, \chi_{n} \perp \mathcal{L}_{n-1}$ and $\left\langle\chi_{n}, \chi_{n}\right\rangle_{\mu}=1$ for every $n \geq 0$. We now have the following lemma.

Lemma 2.1. Let $\chi_{n}(z)=\frac{P_{n}(z)}{\pi_{n}(z)} \in \mathcal{L}_{n} \backslash \mathcal{L}_{n-1}$. Then $P_{n}$ has exactly $n$ zeros in $\mathbb{D}:=\{z \in \mathbb{C}:|z|<1\}$.

Proof. Since all the zeros of $\chi_{n}$ are in $\mathbb{D}$ because of [5. Corollary 3.2.2(3)], a zero of $P_{n}$ that is not a zero of $\chi_{n}$ is only possible if it cancels a zero of $\pi_{n}$. Suppose there exists an $\alpha \in \mathbb{D}$ such that $1 / \bar{\alpha}$ is a zero of $P_{n}$ with multiplicity $m \geq 1$. For the special case in which $\alpha=0$, we say that $P_{n}$ has a zero at infinity with multiplicity $m$ iff $P_{n} \in \mathbb{P}_{n-m}$. Hence it follows that there are at least $m$ indices $j \in\{1, \ldots, n\}$ for which $\alpha_{j}=\alpha$.

Whatever the choice of the sequence of $\alpha_{k}$ is, it should always hold that $\chi_{n} \in$ $\mathcal{L}_{n} \backslash \mathcal{L}_{n-1}$. Now take all $\alpha_{k}$ that are different from $\alpha$ fixed, and consider $\alpha$ variable. Note that $\chi_{n}$ depends continuously on $\alpha$, as do $P_{n}$ and $\pi_{n}$. So let us make this explicit by writing $\chi_{n}(z, \alpha)=P_{n}(z, \alpha) / \pi_{n}(z, \alpha)$. Since $P_{n}(z, \alpha)$ is of the form

$$
P_{n}(z, \alpha)=(1-\bar{\alpha} z)^{m} \hat{P}_{n-m}(z, \alpha) \quad \text { with } \quad \hat{P}_{n-m} \in \mathbb{P}_{n-m},
$$

\footnotetext{
${ }^{2}$ In literature, this is also referred to as the reversed or reciprocal polynomial.
} 
we obtain for $\alpha \rightarrow \alpha_{n}$,

$$
\begin{aligned}
\chi_{n}\left(z, \alpha_{n}\right) & =\lim _{\alpha \rightarrow \alpha_{n}} \frac{(1-\bar{\alpha} z)^{m} \hat{P}_{n-m}(z, \alpha)}{\pi_{n}(z, \alpha)} \\
& =\frac{\left(1-\overline{\alpha_{n}} z\right)^{m-1} \hat{P}_{n-m}\left(z, \alpha_{n}\right)}{\pi_{n-1}\left(z, \alpha_{n}\right)} \in \mathcal{L}_{n-1},
\end{aligned}
$$

contradicting our assumption that $\chi_{n}(z, \alpha) \in \mathcal{L}_{n} \backslash \mathcal{L}_{n-1}$ for all possible choices of $\alpha \in \mathbb{D}$.

Next, consider for every $n, p, q \geq 0$ the sets

$$
\begin{gathered}
\mathcal{L}_{n *}=\left\{f: f_{*} \in \mathcal{L}_{n}\right\} \\
\mathcal{L}_{p, q}=\mathcal{L}_{q} \cdot \mathcal{L}_{p *}=\left\{f g: f \in \mathcal{L}_{q}, g \in \mathcal{L}_{p *}\right\}=\left\{\frac{P(z)}{\pi_{q}(z) \pi_{p}^{*}(z)}: P \in \mathbb{P}_{p+q}\right\}
\end{gathered}
$$

and $\mathcal{R}_{n}=\mathcal{L}_{n, n}$. Following the ordinary polynomial situation (see [25]), we say that a sequence of functions $\Psi_{n}(z) \in \mathcal{L}_{n}$ is para-orthogonal whenever $\Psi_{n}(z) \perp \mathcal{L}_{n-1} \cap$ $\mathcal{L}_{n}\left(\alpha_{n}\right)$, where $\mathcal{L}_{n}\left(\alpha_{n}\right)=\left\{f \in \mathcal{L}_{n}: f\left(\alpha_{n}\right)=0\right\}$, and $\left\langle\Psi_{n}(z), 1\right\rangle_{\mu} \cdot\left\langle\Psi_{n}(z), B_{n}(z)\right\rangle_{\mu} \neq$ 0 . Furthermore, $\Psi_{n}(z)$ is called " $\kappa_{n}$-invariant" iff there exists a $\kappa_{n} \in \mathbb{T}$ so that $\Psi_{n}^{*}(z)=\kappa_{n} \Psi_{n}(z)$ for every $z \in \mathbb{C}$. Note that the concept of $\kappa_{n}$-invariance is usually defined in literature for $\kappa_{n} \in \mathbb{C} \backslash\{0\}$. However, there can only exist $\kappa_{n}$-invariant rational functions whenever $\kappa_{n} \in \mathbb{T}$. Indeed, if $\Psi_{n}(z)=\frac{P_{n}(z)}{\pi_{n}(z)} \in \mathcal{L}_{n}$ is $\kappa_{n}$-invariant, then $\Psi_{n}^{*}(z)=\frac{P_{n}^{*}(z)}{\pi_{n}(z)}=\frac{\kappa_{n} P_{n}(z)}{\pi_{n}(z)}$. Consequently, it remains to prove the statement for ordinary polynomials. If $P_{n}(z)=\sum_{k=0}^{n} c_{k} z^{k}$, with $c_{n} \neq 0$, is $\kappa_{n}$-invariant, then it holds that $c_{0}=P(0) \neq 0, \overline{c_{n}}=\kappa_{n} c_{0}$ and $\overline{c_{0}}=\kappa_{n} c_{n}$. Consequently, $\kappa_{n}=\frac{\overline{c_{n}}}{c_{0}}=\frac{\overline{c_{0}}}{c_{n}}$, which implies that $\left|c_{n}\right|=\left|c_{0}\right|$, and hence, that $\kappa_{n} \in \mathbb{T}$.

$\kappa_{n}$-invariant para-orthogonal rational functions for $\mu$ are characterized in [3] as

$$
\Psi_{n}(z)=\Psi_{n}\left(z, \tau_{n}\right)=C_{n}\left[\phi_{n}(z)+\tau_{n} \phi_{n}^{*}(z)\right] \in \mathcal{L}_{n} ; n \geq 1, C_{n} \in \mathbb{C} \backslash\{0\},
$$

where $\tau_{n}=\frac{\overline{C_{n} \kappa_{n}}}{C_{n}} \in \mathbb{T}$, and $\phi_{n}(z)$ is an $n$th orthogonal rational function for $\mu$. Furthermore, it is proved in [7, Theorem 2.4] that it suffices to compute $\phi_{n-1}(z)$ for the computation of $\Psi_{n}(z)$.

The following result, proved by Bultheel et al. (see [5, Chapter 5]), is an extension of a well-known characterization for Szegö quadrature formulas (see 25]) to the rational case.

Theorem 2.2 (Rational Szegő quadrature). Let $n \geq 1$ and $\tau_{n} \in \mathbb{T}$. Then,

(1) $\Psi_{n}\left(z, \tau_{n}\right)$, given by (4), has exactly $n$ distinct zeros on $\mathbb{T}$,

(2) there exist positive numbers $\lambda_{1}, \ldots, \lambda_{n}$ so that

$$
I_{n}(f)=\sum_{j=1}^{n} \lambda_{j} f\left(z_{j}\right)=I_{\mu}(f)=\int_{-\pi}^{\pi} f\left(e^{\mathrm{i} \theta}\right) d \mu(\theta), \forall f \in \mathcal{R}_{n-1},
$$

where $z_{1}, \ldots, z_{n}$ are the zeros of $\Psi_{n}\left(z, \tau_{n}\right)$,

(3) $\mathcal{R}_{n-1}$ is a maximal domain of validity, i.e., there cannot be exactness in $\mathcal{L}_{n-1, n}$, nor in $\mathcal{L}_{n, n-1}$.

A connection between quadrature formulas on the unit circle and the interval $[-1,1]$ is given in [1]. If $\sigma(x)$ is a weight function on $[-1,1]$, we obtain a weight function on $\mathbb{T}$ by setting $\mu^{\prime}(\theta)=\omega(\theta)=\sigma(\cos \theta)|\sin \theta|$ (see [28], where 
$\mu^{\prime}$ denotes the Radon-Nikodym derivative of the measure $\mu$ with respect to the Lebesgue measure. In the special case in which $\sigma(x)$ is a Jacobi weight function, i.e., $\sigma(x)=(1-x)^{\alpha}(1+x)^{\beta}$, with $\alpha, \beta>-1$, the corresponding weight function on $\mathbb{T}$ is given by

$$
\begin{aligned}
\omega(\theta) & =(1-\cos \theta)^{\alpha}(1+\cos \theta)^{\beta}\left|1-\cos ^{2} \theta\right|^{\frac{1}{2}} \\
& =(1-\cos \theta)^{a}(1+\cos \theta)^{b}, a=\alpha+\frac{1}{2}>-\frac{1}{2}, b=\beta+\frac{1}{2}>-\frac{1}{2} .
\end{aligned}
$$

Finally, if $a, b \in\{0,1\}$, the so-called Chebyshev weight functions appear. Therefore, we set

$$
\omega_{1}(\theta) \equiv 1, \quad \omega_{2}(\theta)=\sin ^{2} \theta, \quad \omega_{3}(\theta)=1-\cos \theta, \omega_{4}(\theta)=1+\cos \theta .
$$

In the remainder of this paper we shall be concerned with rational Szegö quadratures associated with the Chebyshev weight functions $\omega(\theta)=\omega_{i}(\theta), i=1, \ldots, 4$ given by (6). We start in the next section with giving explicit expressions for the corresponding ORFs.

\section{ORFs Associated With Chebyshev weight Functions}

As it is known, few measures give rise to explicit expressions for orthogonal polynomials and even less for ORFs; generally, the computation of such a family proceeds by using a recursive process (see e.g. [5, Theorem 4.1.1]).

It is well known that the so-called Malmquist basis, given by

$$
\phi_{0}^{(1)}(z) \equiv 1 \quad \text { and } \quad \phi_{n}^{(1)}(z)=\frac{z B_{n-1}(z)}{1-\overline{\alpha_{n}} z}, n>0,
$$

is an orthogonal basis for the Lebesgue measure $d \mu(\theta)=\omega_{1}(\theta) d \theta \equiv d \theta$ (see e.g. [5. p. 51]). Recently, explicit expressions are derived in [16] for ORFs associated with the weight functions $\omega_{i}(\theta), i \in\{3,4\}$, given by (6). Let $Q_{n}(z)$ be defined as

$$
Q_{n}(z)=1+z \frac{B_{n-1}^{\prime}(z)}{B_{n-1}(z)}=1+\sum_{k=1}^{n-1} \frac{1-\left|\alpha_{k}\right|^{2}}{\left|z-\alpha_{k}\right|^{2}} \quad \text { for } \quad z \in \mathbb{T} \text {. }
$$

We then have the following theorem ([16]).

Theorem 3.1. Let $i \in\{3,4\}$ be fixed and set $\nu_{i}=(-1)^{i-1}$. Next, define

$$
X_{n}^{(i)}(z)=a_{n}^{(i)}+z^{2}\left(z-b_{n}^{(i)}\right) \frac{B_{n-1}(z)}{1-\overline{\alpha_{n}} z},
$$

where

$$
a_{n}^{(i)}=\frac{\nu_{i} B_{n-1}\left(\nu_{i}\right)}{\left(1-\nu_{i} \overline{\alpha_{n}}\right) Q_{n}^{(i)}+1}, \quad b_{n}^{(i)}=\nu_{i}+\frac{1-\nu_{i} \overline{\alpha_{n}}}{B_{n-1}\left(\nu_{i}\right)} a_{n}^{(i)}
$$

and $Q_{n}^{(i)}=Q_{n}\left(\nu_{i}\right)$ (note that this way, $X_{n}^{(i)}\left(\nu_{i}\right)=X_{n}^{(i)^{\prime}}\left(\nu_{i}\right)=0$ ). Then the sequence of rational functions $\left\{\phi_{n}^{(i)}(z)\right\}_{n=0}^{\infty}$, where

$$
\phi_{0}^{(i)}(z) \equiv 1 \quad \text { and } \quad \phi_{n}^{(i)}(z)=\frac{X_{n}^{(i)}(z)}{\left(z-\nu_{i}\right)^{2}}, \quad n>0
$$


forms a set of ORFs with respect to $\omega_{i}(\theta)=1-\nu_{i} \cos \theta$. Furthermore, the sequence $\left\{\chi_{n}^{(i)}(z)\right\}_{n=0}^{\infty}$, with $\chi_{n}^{(i)}(z)=c_{n}^{(i)} \phi_{n}^{(i)}(z)$, where

$$
\left|c_{0}^{(i)}\right|^{2}=\frac{1}{2 \pi} \quad \text { and } \quad\left|c_{n}^{(i)}\right|^{2}=\nu_{i} \frac{\left(1-\left|\alpha_{n}\right|^{2}\right)\left(1-\nu_{i} \alpha_{n}\right)^{2}}{\pi\left(b_{n}^{(i)}-\alpha_{n}\right)\left(1-\alpha_{n} \overline{b_{n}^{(i)}}\right)}, n>0,
$$

forms a set of orthonormal rational functions with respect to $\omega_{i}(\theta)$.

So far, explicit expressions for ORFs associated with the weight function $\omega_{2}(\theta)$ in (6) are still not known (as indicated in 16]). So, in the remainder of this section we will deal with this open problem.

Note that

$$
\begin{aligned}
\omega_{2}(\theta) & =\sin ^{2} \theta=(1+\cos \theta)(1-\cos \theta)=\left(1+\frac{z^{2}+1}{2 z}\right)(1-\cos \theta) \\
& =\frac{1}{2}|z+1|^{2}(1-\cos \theta)=\frac{1}{2}|z+1|^{2} \omega_{3}(\theta), \quad z=e^{\mathbf{i} \theta} .
\end{aligned}
$$

Hence, suppose $\phi_{n+1}^{(3)}$ is a rational function with poles in $\left\{0, \alpha_{1}, \ldots, \alpha_{n}\right\}$ that is orthogonal on the unit circle with respect to the weight function $\omega_{3}(\theta)$. Further, let $\phi_{n}^{(2)}$ be a rational function with poles in $\left\{\alpha_{1}, \ldots, \alpha_{n}\right\}$ that is orthogonal on the unit circle with respect to the weight function $\omega_{2}(\theta)$. Then for $n>0$, it follows from [14, Theorem 6] that there exist constants $u_{n}, t_{n}$ and $v_{n}$ so that

$$
(z+1)^{2} \phi_{n}^{(2)}(z)=\left(u_{n} z+t_{n}\right) \phi_{n+1}^{(3)}(z)+v_{n}\left(1-\overline{\alpha_{n}} z\right) \phi_{n+1}^{(3) *}(z) .
$$

We now have the following two theorems.

Theorem 3.2. Suppose

$$
\begin{array}{llll}
a^{+}= & \frac{B_{n-1}(1)}{1-\overline{\alpha_{n}}}, & a^{-}= & \frac{B_{n-1}(-1)}{1+\overline{\alpha_{n}}}, \\
b^{+}= & 1+Q_{n}^{(3)}+\frac{1}{1-\overline{\alpha_{n}}}, & b^{-}= & 1+Q_{n}^{(4)}+\frac{1}{1+\overline{\alpha_{n}}},
\end{array}
$$

where $Q_{n}^{(3)}$ and $Q_{n}^{(4)}$ are defined as before in Theorem 3.1, Next, let

$$
\underline{x}=\left(d_{n}, e_{n}, f_{n}, g_{n}\right)^{T}, \quad \underline{y}=\left(-a^{+}, a^{-},-a^{+}\left(b^{+}+2\right), a^{-}\left(b^{-}+2\right)\right)^{T}
$$

and

$$
\boldsymbol{A}=\left(\begin{array}{cccc}
1 & 1 & a^{+} & a^{+} \\
1 & -1 & -a^{-} & a^{-} \\
0 & 1 & a^{+} b^{+} & a^{+}\left(b^{+}+1\right) \\
0 & -1 & -a^{-} b^{-} & a^{-}\left(b^{-}+1\right)
\end{array}\right)
$$

and assume

$$
\underline{x}=A^{-1} \underline{y}=\frac{1}{\varrho_{n}}\left(\begin{array}{c}
4 a^{+} a^{-}\left(b^{+} a^{-}-b^{-} a^{+}\right) \\
-4 a^{+} a^{-}\left[a^{+}\left(b^{-}+1\right)+a^{-}\left(b^{+}+1\right)\right] \\
\left(a^{+}+a^{-}\right)^{2}+4 a^{+} a^{-}\left(b^{+}+b^{-}+b^{+} b^{-}\right) \\
-2\left[2 a^{+} a^{-}\left(b^{+}-b^{-}\right)+\left(a^{+}+a^{-}\right)\left(a^{+}-a^{-}\right)\right]
\end{array}\right),
$$

where $\varrho_{n}$ is given by

$$
\varrho_{n}=\left(a^{+}+a^{-}\right)^{2}-4 a^{+} a^{-} b^{+} b^{-} .
$$

Define $X_{n}^{(2)}$ by

$$
X_{n}^{(2)}(z)=d_{n}+e_{n} z+z^{3}\left(f_{n}+g_{n} z+z^{2}\right) \frac{B_{n-1}(z)}{1-\overline{\alpha_{n}} z},
$$


and set

$$
\phi_{0}^{(2)}(z) \equiv 1 \quad \text { and } \quad \phi_{n}^{(2)}(z)=\frac{X_{n}^{(2)}(z)}{\left(z^{2}-1\right)^{2}} .
$$

Then the sequence of rational functions $\left\{\phi_{n}^{(2)}(z)\right\}_{n=0}^{\infty}$ forms a set of ORFs with respect to $\omega_{2}(\theta)=\sin ^{2} \theta$.

Proof. (The computations are cumbersome; therefore, we will only give the outline of the proof.) From Theorem 3.1 and (13) it follows that for $n>0, \phi_{n}^{(2)}(z)$ should be of the form

$$
\phi_{n}^{(2)}(z)=C_{n} \frac{X_{n}^{(2)}(z)}{\left(z^{2}-1\right)^{2}}, \quad C_{n} \neq 0
$$

where $X_{n}^{(2)}$ is given by (17). For the sake of simplicity, we may as well assume that $C_{n}=1$. Furthermore, we should have that $\phi_{n}^{(2)} \in \mathcal{L}_{n}$. Consequently, it must hold that $X_{n}^{(2)}( \pm 1)=X_{n}^{(2)^{\prime}}( \pm 1)=0$. This leaves us with a system of four equations in the four unknowns $d_{n}, e_{n}, f_{n}$ and $g_{n}$. Solving this system for the coefficients $d_{n}, e_{n}, f_{n}$ and $g_{n}$ then gives (15). The analytic solution of (15), given by the second equality in (15) and (16), has been computed with the aid of Maple 1(3.

Theorem 3.3. The sequence $\left\{\chi_{n}^{(2)}(z)\right\}_{n=0}^{\infty}$, with $\chi_{n}^{(2)}(z)=h_{n} \phi_{n}^{(2)}(z)$, where

$$
\left|h_{0}\right|^{2}=\frac{1}{\pi}, \quad\left|h_{n}\right|^{2}=\frac{-2\left(1-\left|\alpha_{n}\right|^{2}\right)\left(1-\alpha_{n}^{2}\right)^{2}}{\pi\left(f_{n}+g_{n} \alpha_{n}+\alpha_{n}^{2}\right)\left(\overline{f_{n}} \alpha_{n}^{2}+\overline{g_{n}} \alpha_{n}+1\right)}, \quad n>0,
$$

and $\phi_{n}^{(2)}(z)$ is defined as before in Theorem 3.2, forms a set of orthonormal rational functions with respect to $\omega_{2}(\theta)=\sin ^{2} \theta$.

Proof. The expression for $n=0$ is easily verified; so, we continue for $n>0$. First, note that

$$
\sin ^{2}(\theta) d \theta=-\left(z^{2}-1\right)^{2} \frac{d z}{4 \mathbf{i} z^{3}}, \quad z=e^{\mathbf{i} \theta} .
$$

Hence, we have that

$$
\begin{aligned}
\left\langle\chi_{n}^{(2)}, B_{n}\right\rangle_{\omega_{2}} & =-\frac{1}{4 \mathbf{i}} \int_{\mathbb{T}} \chi_{n}^{(2)}(z) B_{n *}(z)\left(z^{2}-1\right)^{2} \frac{d z}{z^{3}} \\
& =-\frac{h_{n}}{4 \mathbf{i}}\left[\int_{\mathbb{T}} \frac{\left(d_{n}+e_{n} z\right) B_{n *}(z)}{z^{3}} d z+\int_{\mathbb{T}} \frac{\left(f_{n}+g_{n} z+z^{2}\right) B_{n-1}(z)}{\left(1-\overline{\alpha_{n}} z\right) B_{n}(z)} d z\right] \\
& =-\frac{h_{n}}{4 \mathbf{i}}\left[-\int_{\mathbb{T}} \frac{\left(d_{n}+e_{n} \bar{z}\right) B_{n *}(\bar{z})}{\bar{z}^{3}} d \bar{z}+\int_{\mathbb{T}} \frac{f_{n}+g_{n} z+z^{2}}{z-\alpha_{n}} d z\right] \\
& =-\frac{h_{n}}{4 \mathbf{i}}\left[\int_{\mathbb{T}}\left(d_{n} z+e_{n}\right) \overline{B_{n}(\bar{z})} d z+\int_{\mathbb{T}} G_{n}(z) d z\right] \\
& =-\frac{h_{n}}{4 \mathbf{i}}\left[\int_{\mathbb{T}} F_{n}(z) d z+\int_{\mathbb{T}} G_{n}(z) d z\right] .
\end{aligned}
$$

\footnotetext{
${ }^{3}$ Maple and Maple V are registered trademarks of Waterloo Maple, Inc.
} 
Clearly, $F_{n}$ is analytic in $\mathbb{D}:=\{z \in \mathbb{C}:|z|<1\}$, so that

$$
\begin{aligned}
\left\langle\chi_{n}^{(2)}, B_{n}\right\rangle_{\omega_{2}} & =-\frac{h_{n}}{4 \mathbf{i}} \int_{\mathbb{T}} G_{n}(z) d z=-\frac{h_{n}}{4 \mathbf{i}} \int_{\mathbb{T}} \frac{f_{n}+g_{n} z+z^{2}}{z-\alpha_{n}} d z \\
& =-\frac{h_{n} \pi}{2} \operatorname{Res}\left\{\frac{f_{n}+g_{n} z+z^{2}}{z-\alpha_{n}}, \alpha_{n}\right\}=-\frac{h_{n} \pi\left(f_{n}+g_{n} \alpha_{n}+\alpha_{n}^{2}\right)}{2} .
\end{aligned}
$$

Finally, suppose $\chi_{n}^{(2)}$ is of the form

$$
\chi_{n}^{(2)}(z)=\sum_{k=0}^{n} a_{k} B_{k}(z) .
$$

Then it is easily verified that $\left\langle\chi_{n}^{(2)}, B_{n}\right\rangle_{\omega_{2}}=\frac{1}{\overline{a_{n}}}$, with

$$
\overline{a_{n}}=\chi_{n}^{(2) *}\left(\alpha_{n}\right)=\frac{\overline{h_{n}}\left(\overline{f_{n}} \alpha_{n}^{2}+\overline{g_{n}} \alpha_{n}+1\right)}{\left(1-\left|\alpha_{n}\right|^{2}\right)\left(1-\alpha_{n}^{2}\right)^{2}} .
$$

Consequently,

$$
\left|h_{n}\right|^{2}=-\frac{2\left(1-\left|\alpha_{n}\right|^{2}\right)\left(1-\alpha_{n}^{2}\right)^{2}}{\pi\left(f_{n}+g_{n} \alpha_{n}+\alpha_{n}^{2}\right)\left(\overline{f_{n}} \alpha_{n}^{2}+\overline{g_{n}} \alpha_{n}+1\right)},
$$

which ends the proof.

Finally, we will also need the following lemma.

Lemma 3.4. It holds that

$$
d_{n}+e_{n} \epsilon \neq 0
$$

where $\epsilon \in\{ \pm 1\}$, and $d_{n}$ and $e_{n}$ are defined as before in Theorem 3.2 ,

Proof. Suppose $d_{n}+e_{n} \epsilon=0$ for a fixed $\epsilon \in\{ \pm 1\}$. We then have that

$$
d_{n}+e_{n} z=e_{n}(z-\epsilon) \text {. }
$$

Since $a^{+}$and $a^{-}$, given by (14), are different from zero, and $X_{n}^{(2)}(\epsilon)=0$, we also have that

$$
\left(f_{n}+g_{n} z+z^{2}\right)=(z-\epsilon)\left(z-f_{n} \epsilon\right) .
$$

Consequently,

$$
X_{n}^{(2)}(z)=(z-\epsilon)\left[e_{n}+z^{3}\left(z-f_{n} \epsilon\right) \frac{B_{n-1}(z)}{1-\overline{\alpha_{n}} z}\right]=(z-\epsilon) \hat{X}_{n}(z) .
$$

Note that for $z \in \mathbb{T}$ it holds that

$$
\hat{X}_{n}^{\prime}(z)=z^{2} \frac{B_{n-1}(z)}{1-\overline{\alpha_{n}} z}\left[z\left(2+Q_{n}(z)+\frac{1}{1-\overline{\alpha_{n}}}\right)-f_{n} \epsilon\left(1+Q_{n}(z)+\frac{1}{1-\overline{\alpha_{n}}}\right)\right],
$$

where $Q_{n}(z)$ is defined as before in (8), so that

$$
\begin{aligned}
\left\{\begin{array}{l}
X_{n}^{(2)^{\prime}}(1)=0 \\
X_{n}^{(2)^{\prime}}(-1)=0
\end{array}\right. & \Leftrightarrow\left\{\begin{array} { l } 
{ \hat { X } _ { n } ^ { \prime } ( 1 ) = 0 } \\
{ \hat { X } _ { n } ^ { \prime } ( - 1 ) = 0 }
\end{array} \Leftrightarrow \left\{\begin{array}{l}
a^{+}\left[\left(1+b^{+}\right)-f_{n} \epsilon b^{+}\right]=0 \\
a^{-}\left[\left(1+b^{-}\right)+f_{n} \epsilon b^{-}\right]=0
\end{array}\right.\right. \\
& \Leftrightarrow\left\{\begin{array} { l } 
{ f _ { n } \epsilon = 1 + \frac { 1 } { b ^ { + } } } \\
{ f _ { n } \epsilon = - ( 1 + \frac { 1 } { b ^ { - } } ) }
\end{array} \Leftrightarrow \left\{\begin{array}{l}
f_{n} \epsilon=1+\frac{1}{b^{+}} \\
\frac{1}{b^{+}}+\frac{1}{b^{-}}=-2
\end{array}\right.\right.
\end{aligned}
$$


Furthermore, since $\alpha_{n} \in \mathbb{D}$, we have that $0<\Re\left\{\frac{1}{1 \pm \alpha_{n}}\right\}<\infty$. From (8) and (14) we then deduce that $2<\Re\left\{b^{+}\right\}, \Re\left\{b^{-}\right\}<\infty$, so that

$$
-2=\Re\left\{\frac{1}{b^{+}}+\frac{1}{b^{-}}\right\}=\Re\left\{\frac{1}{b^{+}}\right\}+\Re\left\{\frac{1}{b^{-}}\right\}>0 .
$$

This is a contradiction, and hence, $d_{n}+e_{n} \epsilon \neq 0$.

\section{Rational Szegö quadratures associated with Chebyshev weight functions}

The aim of this section is to characterize rational Szegö formulas $I_{n}(f)$, given by (5), associated with the Chebyshev weight functions $\omega_{i}(\theta), i=1, \ldots, 4$, given by (6). Therefore, we will derive explicit expressions for the associated paraorthogonal rational functions $\Psi_{n}\left(z, \tau_{n}\right)$ by means of (4) along with the results provided in the previous section. The zeros $\left\{z_{j}\right\}_{j=1}^{n}$ of $\Psi_{n}\left(z, \tau_{n}\right)$ are the nodes we need for $I_{n}(f)$. First, let us consider the reproducing kernel function for $\mathcal{L}_{n}$ associated with a general measure $\mu$, namely

$$
\mathcal{K}_{n}(z, \xi)=\sum_{k=0}^{n} \chi_{k}(z) \overline{\chi_{k}(\xi)}
$$

The following Christoffel-Darboux formula has been proved in [5, Theorem 3.1.3]:

$$
\mathcal{K}_{n}(z, \xi)=\frac{\chi_{n+1}^{*}(z) \overline{\chi_{n+1}^{*}(\xi)}-\chi_{n+1}(z) \overline{\chi_{n+1}(\xi)}}{1-\zeta_{n+1}(z) \overline{\zeta_{n+1}(\xi)}}, n \geq 1 .
$$

Moreover, the following well-known expression for the weights in a Szegó quadrature formula has been proved for the rational case in [5, Theorem 5.4.2]:

$$
\lambda_{j}=\frac{1}{\sum_{k=0}^{n-1}\left|\chi_{k}\left(z_{j}\right)\right|^{2}} ; j=1, \ldots, n .
$$

We are now able to prove the following proposition.

Proposition 4.1. Let $\left\{z_{j}\right\}_{j=1}^{n}$ and $\left\{\lambda_{j}\right\}_{j=1}^{n}$ represent the set of nodes and weights of a rational Szegö quadrature formula (5) for $\mu$, and suppose $\chi_{n}(z)$ is the corresponding nth orthonormal rational function. It then holds that

$$
\lambda_{j}^{-1}=\frac{z_{j}\left|z_{j}-\alpha_{n}\right|^{2}}{1-\left|\alpha_{n}\right|^{2}}\left|\begin{array}{cc}
\overline{\chi_{n}\left(z_{j}\right)} & \overline{\chi_{n}^{*}\left(z_{j}\right)} \\
\left(\chi_{n}^{*}\right)^{\prime}\left(z_{j}\right) & \chi_{n}^{\prime}\left(z_{j}\right)
\end{array}\right|, j=1, \ldots, n .
$$

Proof. From (19) and (20) it follows that

$$
\lambda_{j}^{-1}=\lim _{\substack{z \rightarrow z_{j} \\ z \in \mathbb{T}}} \mathcal{K}_{n-1}\left(z, z_{j}\right)=\frac{\left(\chi_{n}^{*}\right)^{\prime}\left(z_{j}\right) \overline{\chi_{n}^{*}\left(z_{j}\right)}-\chi_{n}^{\prime}\left(z_{j}\right) \overline{\chi_{n}\left(z_{j}\right)}}{-\zeta_{n}^{\prime}\left(z_{j}\right) \overline{\zeta_{n}\left(z_{j}\right)}} .
$$

The statement is now easily verified by taking into account that $\overline{\zeta_{n}(z)}=1 / \zeta_{n}(z)$ and $\zeta_{n}^{\prime}(z)=\frac{1-\left|\alpha_{n}\right|^{2}}{z\left|z-\alpha_{n}\right|^{2}} \zeta_{n}(z)$ for $z \in \mathbb{T}$.

We start with the Lebesgue measure $d \mu(\theta)=\omega_{1}(\theta) d \theta \equiv d \theta$. From (4) and (7) it follows that, up to a multiplicative factor,

$$
\Psi_{n}^{(1)}\left(z, \tau_{n}\right)=\frac{V_{n}^{(1)}\left(z, \tau_{n}\right)}{\pi_{n}(z)}, \quad z, \tau_{n} \in \mathbb{T},
$$


where

$$
V_{n}^{(1)}\left(z, \tau_{n}\right)=p_{0}^{(1)}(z) \pi_{n-1}(z)+\tau_{n} z p_{0}^{(1) *}(z) \pi_{n-1}^{*}(z)
$$

and

$$
p_{0}^{(1)}(z) \equiv \tau_{n}
$$

Moreover, for this special case it is well known that (see [4])

$$
\lambda_{j}^{(1)}=\frac{2 \pi}{Q_{n}\left(z_{j}^{(1)}\right)}, j=1, \ldots, n
$$

with $Q_{n}(z)$ given by (지 $)$. Indeed, let $\chi_{n}^{(1)}(z)=k_{n} \phi_{n}^{(1)}(z)$ with $\phi_{n}^{(1)}(z)$ given by (17) and $\left|k_{n}\right|^{2}=\frac{1-\left|\alpha_{n}\right|^{2}}{2 \pi}$ for $n \geq 1$. Then it follows from Proposition 4.1 that

$$
\begin{aligned}
& \lambda_{j}^{(1)}=\frac{1-\left|\alpha_{n}\right|^{2}}{z_{j}^{(1)}\left|z_{j}^{(1)}-\alpha_{n}\right|^{2}}
\end{aligned}
$$

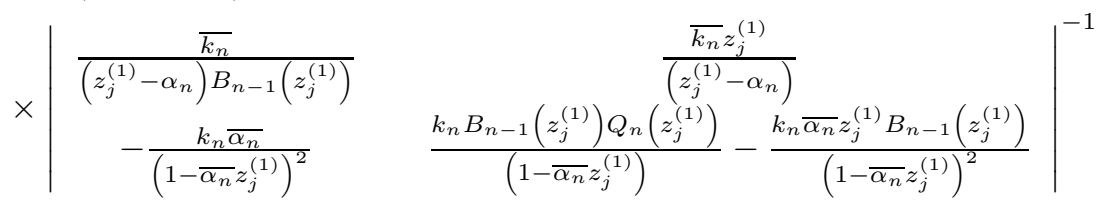

$$
\begin{aligned}
& =\frac{1-\left|\alpha_{n}\right|^{2}}{z_{j}^{(1)}\left|z_{j}^{(1)}-\alpha_{n}\right|^{2}} \times\left[\frac{\left|k_{n}\right|^{2} Q_{n}\left(z_{j}^{(1)}\right)}{\left(z_{j}^{(1)}-\alpha_{n}\right)\left(1-\overline{\alpha_{n}} z_{j}^{(1)}\right)}\right]^{-1}=\frac{1-\left|\alpha_{n}\right|^{2}}{\left|k_{n}\right|^{2} Q_{n}\left(z_{j}^{(1)}\right)} .
\end{aligned}
$$

Thus, we have the following theorem.

Theorem 4.2. Let $n \geq 1$ and $\tau_{n} \in \mathbb{T}$. The nodes of an $n$-point rational Szegö quadrature $I_{n}(f)$ for $I_{\omega_{1}}(f)$, given by (5) and (6), are then the zeros of $V_{n}^{(1)}\left(z, \tau_{n}\right)$ given by (23) and (24), while the weights are given by (25).

Next, consider the weight functions $\omega_{i}(\theta)=1-\nu_{i} \cos \theta$, with $i \in\{3,4\}$ and $\nu_{i}=(-1)^{i-1}$. From (4) and Theorem 3.1 it follows that, up to a multiplicative constant,

$$
\Psi_{n}^{(i)}\left(z, \tau_{n}\right)=\frac{V_{n}^{(i)}\left(z, \tau_{n}\right)}{\left(z-\nu_{i}\right)^{2} \pi_{n}(z)}, \quad i=3,4, \quad z, \tau_{n} \in \mathbb{T}
$$

where

$$
V_{n}^{(i)}\left(z, \tau_{n}\right)=p_{1}^{(i)}(z) \pi_{n-1}(z)+\tau_{n} z^{2} p_{1}^{(i) *}(z) \pi_{n-1}^{*}(z)
$$

and

$$
p_{1}^{(i)}(z)=a_{n}^{(i)}\left(1-\overline{\alpha_{n}} z\right)+\tau_{n}\left(1-\overline{b_{n}^{(i)}} z\right) .
$$

Explicit expressions for the weights can be deduced as well from Theorem 3.1 and (21). Suppose $z_{j}=z_{j}^{(i)} \neq \nu_{i}$ for every $j \in\{1, \ldots, n\}$ and fixed $i \in\{3,4\}$. Next, let

$$
T_{n}^{(i)}=\overline{X_{n}^{(i)}\left(z_{j}\right)}\left[\left(Q_{n+1}\left(z_{j}\right)+1\right)\left(1-\nu_{i} z_{j}\right)+2 \nu_{i} z_{j}\right]+z_{j}\left(\overline{X_{n}^{(i)}\left(z_{j}\right)}\right)^{\prime}\left(1-\nu_{i} z_{j}\right) .
$$


Then, with $\lambda_{j}=\lambda_{j}^{(i)}$ and $\left|c_{n}^{(i)}\right|^{2}$ given by (12), it follows that

$$
\begin{aligned}
& \lambda_{j}^{-1}=\left|c_{n}^{(i)}\right|^{2} \frac{z_{j}\left|z_{j}-\alpha_{n}\right|^{2}}{1-\left|\alpha_{n}\right|^{2}}\left|\begin{array}{cc}
\overline{\phi_{n}^{(i)}\left(z_{j}\right)} & \overline{\phi_{n}^{(i) *}\left(z_{j}\right)} \\
\left(\phi_{n}^{(i) *}\right)^{\prime}\left(z_{j}\right) & \phi_{n}^{(i)^{\prime}}\left(z_{j}\right)
\end{array}\right| \\
& =\left|c_{n}^{(i)}\right|^{2} \frac{z_{j}\left|z_{j}-\alpha_{n}\right|^{2}}{1-\left|\alpha_{n}\right|^{2}}\left|\begin{array}{cc}
\frac{z_{j}^{2}}{\left(1-\nu_{i} z_{j}\right)^{2}} \overline{X_{n}^{(i)}\left(z_{j}\right)} & \frac{X_{n}^{(i)}\left(z_{j}\right)}{\bar{B}_{n}\left(z_{j}\right)\left(z_{j}-\nu_{i}\right)^{2}} \\
\frac{z_{j} B_{n}\left(z_{j}\right)}{\left(1-\nu_{i} z_{j}\right)^{3}} T_{n}^{(i)} & \frac{X_{n}^{(i)}\left(z_{j}\right)}{\left(z_{j}-\nu_{i}\right)^{2}}-\frac{2 X_{n}^{(i)}\left(z_{j}\right)}{\left(z_{j}-\nu_{i}\right)^{3}}
\end{array}\right| \\
& =\left|c_{n}^{(i)}\right|^{2} \frac{z_{j}\left|z_{j}-\alpha_{n}\right|^{2}}{\left(1-\left|\alpha_{n}\right|^{2}\right)\left(z_{j}-\nu_{i}\right)^{4}}\left|\begin{array}{cc}
z_{j}^{2} \overline{X_{n}^{(i)}\left(z_{j}\right)} & \frac{X_{n}^{(i)}\left(z_{j}\right)}{B_{n}\left(z_{j}\right)} \\
\frac{z_{j} B_{n}\left(z_{j}\right)}{1-\nu_{i} z_{j}} T_{n}^{(i)} & X_{n}^{(i)^{\prime}}\left(z_{j}\right)-\frac{2 X_{n}^{(i)}\left(z_{j}\right)}{z_{j}-\nu_{i}}
\end{array}\right| \\
& =\left|c_{n}^{(i)}\right|^{2} \frac{\left|z_{j}-\alpha_{n}\right|^{2}}{\left(1-\left|\alpha_{n}\right|^{2}\right)\left|z_{j}-\nu_{i}\right|^{4}} \\
& \times\left\{z_{j}\left[\overline{X_{n}^{(i)}\left(z_{j}\right)} X_{n}^{(i)^{\prime}}\left(z_{j}\right)-X_{n}^{(i)}\left(z_{j}\right)\left(\overline{X_{n}^{(i)}\left(z_{j}\right)}\right)^{\prime}\right]\right. \\
& \left.-\left|X_{n}^{(i)}\left(z_{j}\right)\right|^{2}\left(Q_{n+1}\left(z_{j}\right)+1\right)\right\} \\
& =\left|c_{n}^{(i)}\right|^{2} \frac{\left|z_{j}-\alpha_{n}\right|^{2}}{\left(1-\left|\alpha_{n}\right|^{2}\right)\left|z_{j}-\nu_{i}\right|^{4}} \\
& \times\left\{2 \Re\left[z_{j} \overline{X_{n}^{(i)}\left(z_{j}\right)} X_{n}^{(i)^{\prime}}\left(z_{j}\right)\right]-\left|X_{n}^{(i)}\left(z_{j}\right)\right|^{2}\left(Q_{n+1}\left(z_{j}\right)+1\right)\right\} .
\end{aligned}
$$

Here, the last equality is due to the fact that for $z \in \mathbb{T}$, and for every $f(z)$ that is analytic in a small annulus containing the complex unit circle, it holds that (see e.g. [31, Lem. 3.3])

$$
(\overline{f(z)})^{\prime}=\frac{d \bar{f}}{d z}=\frac{d \bar{f}}{d \bar{z}} \cdot \frac{d \bar{z}}{d z}=-\frac{\overline{f^{\prime}(z)}}{z^{2}}
$$

From (9) we deduce that

$$
X_{n}^{(i)^{\prime}}(z)=\frac{z^{2}\left(z-b_{n}^{(i)}\right) B_{n-1}(z)}{1-\overline{\alpha_{n}} z}\left[\frac{2}{z}+\frac{B_{n-1}^{\prime}(z)}{B_{n-1}(z)}+\frac{1}{z-b_{n}^{(i)}}+\frac{\overline{\alpha_{n}}}{1-\overline{\alpha_{n}} z}\right]
$$

so that for $z \in \mathbb{T}$ it holds that

$$
\begin{array}{r}
\text { (29) } z X_{n}^{(i)^{\prime}}(z)=\left(X_{n}^{(i)}(z)-a_{n}^{(i)}\right)\left(1+Q_{n}(z)+\frac{z}{z-b_{n}^{(i)}}+\frac{\overline{\alpha_{n}} z}{1-\overline{\alpha_{n}} z}\right) \\
=\left(X_{n}^{(i)}(z)-a_{n}^{(i)}\right)\left(1+Q_{n+1}(z)+\frac{z\left(b_{n}^{(i)}-\alpha_{n}\right)}{\left(z-b_{n}^{(i)}\right)\left(z-\alpha_{n}\right)}\right) .
\end{array}
$$


Consequently,

$$
\begin{aligned}
& \lambda_{j}^{-1}=\left|c_{n}^{(i)}\right|^{2} \frac{\left|z_{j}-\alpha_{n}\right|^{2}}{\left(1-\left|\alpha_{n}\right|^{2}\right)\left|z_{j}-\nu_{i}\right|^{4}} \\
& \times\left[\left(\left|X_{n}^{(i)}\left(z_{j}\right)\right|^{2}-2 \Re\left\{a_{n}^{(i)} \overline{X_{n}^{(i)}\left(z_{j}\right)}\right\}\right)\left[1+Q_{n+1}\left(z_{j}\right)\right]\right. \\
&\left.+2 \Re\left\{\frac{z_{j}\left(b_{n}^{(i)}-\alpha_{n}\right)\left(\left|X_{n}^{(i)}\left(z_{j}\right)\right|^{2}-a_{n}^{(i)} \overline{X_{n}^{(i)}\left(z_{j}\right)}\right)}{\left(z_{j}-b_{n}^{(i)}\right)\left(z_{j}-\alpha_{n}\right)}\right\}\right] .
\end{aligned}
$$

Finally, we have that

$$
\begin{aligned}
& \left|X_{n}^{(i)}(z)\right|^{2}-2 \Re\left\{a_{n}^{(i)} \overline{X_{n}^{(i)}(z)}\right\}=\left|\frac{z-b_{n}^{(i)}}{z-\alpha_{n}}\right|^{2}-\left|a_{n}^{(i)}\right|^{2} \\
& \left|X_{n}^{(i)}(z)\right|^{2}-a_{n}^{(i)} \overline{X_{n}^{(i)}(z)}=\left|\frac{z-b_{n}^{(i)}}{z-\alpha_{n}}\right|^{2}+\overline{a_{n}^{(i)}} \frac{z^{2}\left(z-b_{n}^{(i)}\right) B_{n-1}(z)}{1-\overline{\alpha_{n}} z}
\end{aligned}
$$

and

$$
z_{j}^{2} B_{n-1}\left(z_{j}\right)=-\overline{\tau_{n}} \frac{p_{1}^{(i)}\left(z_{j}\right)}{p_{1}^{(i) *}\left(z_{j}\right)}
$$

where $p_{1}^{(i)}(z)$ is given by (27), so that

$$
\begin{aligned}
\lambda_{j}^{-1}=\frac{\left|c_{n}^{(i)}\right|^{2}}{\left(1-\left|\alpha_{n}\right|^{2}\right)\left|z_{j}-\nu_{i}\right|^{4}} & {\left[\left(\left|z_{j}-b_{n}^{(i)}\right|^{2}-\left|a_{n}^{(i)}\right|^{2}\left|z_{j}-\alpha_{n}\right|^{2}\right)\left[1+Q_{n+1}\left(z_{j}\right)\right]\right.} \\
+2 \Re & \left\{\left(b_{n}^{(i)}-\alpha_{n}\right)\left(\frac{1-\overline{b_{n}^{(i)}} z_{j}}{z_{j}-\alpha_{n}}-\overline{a_{n}^{(i)}} \overline{\tau_{n}} \frac{p_{1}^{(i)}\left(z_{j}\right)}{p_{1}^{(i) *}\left(z_{j}\right)}\right)\right\} .
\end{aligned}
$$

Whenever $z_{j}=\nu_{i}$ for a certain $j \in\{1, \ldots, n\}$, computing $\lambda_{j}$ by the aid of (21) requires two times the application of l'Hôpital's rule to compute $\overline{\chi_{n}^{(i)}\left(\nu_{i}\right)}, \overline{\chi_{n}^{(i) *}\left(\nu_{i}\right)}$, $\left(\chi_{n}^{(i) *}\right)^{\prime}\left(\nu_{i}\right)$ and $\chi_{n}^{(i)^{\prime}}\left(\nu_{i}\right)$. This gives rise to tedious calculations along with an inappropriate expression for computational purposes. Since $z_{j} \neq z_{k}$ for $j \neq k$, there can be at most one index $j$ for which $z_{j}=\nu_{i}$. Therefore, $\lambda_{j}$ can then be computed as follows:

$$
\lambda_{j}=\int_{-\pi}^{\pi} \omega_{i}(\theta) d \theta-\sum_{\substack{k=1 \\ k \neq j}}^{n} \lambda_{k}=2 \pi-\sum_{\substack{k=1 \\ k \neq j}}^{n} \lambda_{k} .
$$

\footnotetext{
${ }^{4}$ There are no $\tau_{n} \in \mathbb{T}$ and $\gamma \in \mathbb{T} \backslash\left\{\nu_{i}\right\}$ so that $p_{1}^{(i) *}(\gamma)=0$; hence, the right-hand side of (30) is well defined. A proof for this statement is given in the Appendix.
} 
Note that there exists an index $j$ so that $z_{j}=\nu_{i}$ iff $\tau_{n}=-\frac{\phi_{n}^{(i)}\left(\nu_{i}\right)}{\phi_{n}^{(i) *}\left(\nu_{i}\right)}$. From (11) and (28) it follows that

$$
\begin{aligned}
& -\frac{\phi_{n}^{(i)}\left(\nu_{i}\right)}{\phi_{n}^{(i) *}\left(\nu_{i}\right)}=\frac{-1}{B_{n}\left(\nu_{i}\right)} \lim _{\substack{z \rightarrow \nu_{i} \\
z \in \mathbb{T}}} \frac{X_{n}^{(i)}(z)}{\underline{X_{n}^{(i)}(z)}} \\
& \quad=\frac{1}{B_{n}\left(\nu_{i}\right)} \lim _{\substack{z \rightarrow \nu_{i} \\
z \in \mathbb{T}}} \frac{X_{n}^{(i)^{\prime}}(z)}{X_{n}^{(i)^{\prime}}(z)}=\frac{-1}{B_{n}\left(\nu_{i}\right)} \frac{X_{n}^{(i)^{\prime \prime}}\left(\nu_{i}\right)}{X_{n}^{(i)^{\prime \prime}}\left(\nu_{i}\right)} .
\end{aligned}
$$

Let

$$
Y_{n}^{(i)}(z)=1+Q_{n+1}(z)+\frac{z\left(b_{n}^{(i)}-\alpha_{n}\right)}{\left(z-b_{n}^{(i)}\right)\left(z-\alpha_{n}\right)} .
$$

Then it follows from (29) that

$$
z X_{n}^{(i)^{\prime \prime}}(z)=X_{n}^{(i)^{\prime}}(z)\left(Y_{n}^{(i)}(z)-1\right)+\left(X_{n}^{(i)}(z)-a_{n}^{(i)}\right) Y_{n}^{(i)^{\prime}}(z),
$$

and hence,

$$
\begin{aligned}
X_{n}^{(i)^{\prime \prime}}\left(\nu_{i}\right) & =-a_{n}^{(i)} \nu_{i} Y_{n}^{(i)^{\prime}}\left(\nu_{i}\right)=a_{n}^{(i)}\left[\frac{\nu_{i}\left(b_{n}^{(i)}-\alpha_{n}\right)\left(1-\alpha_{n} b_{n}^{(i)}\right)}{\left(\nu_{i}-b_{n}^{(i)}\right)^{2}\left(\nu_{i}-\alpha_{n}\right)^{2}}-\nu_{i} Q_{n+1}^{\prime}\left(\nu_{i}\right)\right] \\
& =R_{n}^{(i)} e^{\mathbf{i} \gamma_{n}^{(i)}}, \quad R_{n}^{(i)}>0 \text { and } \gamma_{n}^{(i)} \in[0,2 \pi),
\end{aligned}
$$

where

$$
z Q_{n+1}^{\prime}(z)=2 \mathbf{i} \sum_{k=1}^{n} \frac{\Im\left\{\overline{\alpha_{k}} z\right\}\left(1-\left|\alpha_{k}\right|^{2}\right)}{\left|z-\alpha_{k}\right|^{4}} \text { for } z \in \mathbb{T} .
$$

Consequently, there exists an index $j$ so that $z_{j}=\nu_{i}$ iff $\tau_{n}=\frac{e^{\mathbf{i}\left(2 \gamma_{n}^{(i)}-\pi\right)}}{B_{n}\left(\nu_{i}\right)}$.

We now have proved the following theorem.

Theorem 4.3. Let $i \in\{3,4\}$ be fixed and set $\nu_{i}=(-1)^{i-1}$. Further, assume $\tau_{n} \in \mathbb{T}$, for $n \geq 1$. An n-point rational Szegö quadrature $I_{n}(f)$ for $I_{\omega_{i}}(f)$, given by (5) and (6), is then characterized as follows:

(1) Denote by $\left\{\nu_{i}, \nu_{i}, z_{1}, \ldots, z_{n}\right\}$ the set of zeros of $V_{n}^{(i)}\left(z, \tau_{n}\right)$, given by (26) and (27). Then $\left\{z_{1}, \ldots, z_{n}\right\}$ is the set of nodes for $I_{n}(f)$.

(2) For every $j \in\{1, \ldots, n\}$ for which $z_{j} \neq \nu_{i}$, the $j$ th weight is given by (31). Whenever $z_{j}=\nu_{i}$ for a certain $j \in\{1, \ldots, n\}$, the associated weight is given by (32).

To conclude this section, we consider the weight function $\omega_{2}(\theta)$. From (4) and Theorem 3.2 it now follows that, up to a multiplicative factor,

$$
\Psi_{n}^{(2)}\left(z, \tau_{n}\right)=\frac{V_{n}^{(2)}\left(z, \tau_{n}\right)}{\left(z^{2}-1\right)^{2} \pi_{n}(z)}, \quad z, \tau_{n} \in \mathbb{T},
$$

where

$$
V_{n}^{(2)}\left(z, \tau_{n}\right)=p_{2}^{(2)}(z) \pi_{n-1}(z)+\tau_{n} z^{3} p_{2}^{(2) *}(z) \pi_{n-1}^{*}(z)
$$

and

$$
p_{2}^{(2)}(z)=\left(d_{n}+e_{n} z\right)\left(1-\overline{\alpha_{n}} z\right)+\tau_{n}\left(\overline{f_{n}} z^{2}+\overline{g_{n}} z+1\right) .
$$


Explicit expressions for the weights can be deduced as well from (21) and Theorems 3.2 and 3.3. Assume $\epsilon \in\{ \pm 1\}$ and suppose $z_{j}=z_{j}^{(2)} \neq \epsilon$ for every $j \in\{1, \ldots, n\}$. Next, let

$$
T_{n}^{(2)}=\left[Q_{n+1}\left(z_{j}\right)+3-\frac{4 z_{j}^{2}}{z_{j}^{2}-1}\right] z_{j} \overline{X_{n}^{(2)}\left(z_{j}\right)}-\overline{X_{n}^{(2)^{\prime}}\left(z_{j}\right)} .
$$

Then, with $\lambda_{j}=\lambda_{j}^{(2)}$ and $\left|h_{n}\right|^{2}$ given by (18), it now follows that

$$
\begin{aligned}
& \lambda_{j}^{-1}=\left|h_{n}\right|^{2} \frac{z_{j}\left|z_{j}-\alpha_{n}\right|^{2}}{1-\left|\alpha_{n}\right|^{2}}\left|\begin{array}{cc}
\overline{\phi_{n}^{(2)}\left(z_{j}\right)} & \overline{\phi_{n}^{(2) *}\left(z_{j}\right)} \\
\left(\phi_{n}^{(2) *}\right)^{\prime}\left(z_{j}\right) & \phi_{n}^{(2)^{\prime}}\left(z_{j}\right)
\end{array}\right| \\
& =\left|h_{n}\right|^{2} \frac{z_{j}\left|z_{j}-\alpha_{n}\right|^{2}}{1-\left|\alpha_{n}\right|^{2}}\left|\begin{array}{cc}
\frac{z_{j}^{4}}{\left(z_{j}^{2}-1\right)^{2}} \overline{X_{n}^{(2)}\left(z_{j}\right)} & \frac{X_{n}^{(2)}\left(z_{j}\right)}{B_{n}\left(z_{j}\right)\left(z_{j}^{2}-1\right)^{2}} \\
\frac{z_{j}^{2} B_{n}\left(z_{j}\right)}{\left(z_{j}^{2}-1\right)^{2}} T_{n}^{(2)} & \frac{X_{n}^{(2)}\left(z_{j}\right)}{\left(z_{j}^{2}-1\right)^{2}}-\frac{4 z_{j} X_{n}^{(2)}\left(z_{j}\right)}{\left(z_{j}^{2}-1\right)^{3}}
\end{array}\right| \\
& =\left|h_{n}\right|^{2} \frac{z_{j}\left|z_{j}-\alpha_{n}\right|^{2}}{\left(1-\left|\alpha_{n}\right|^{2}\right)\left(z_{j}^{2}-1\right)^{4}}\left|\begin{array}{cc}
z_{j}^{4} \overline{X_{n}^{(2)}\left(z_{j}\right)} & \frac{X_{n}^{(2)}\left(z_{j}\right)}{B_{n}\left(z_{j}\right)} \\
z_{j}^{2} B_{n}\left(z_{j}\right) T_{n}^{(2)} & X_{n}^{(2)^{\prime}}\left(z_{j}\right)-\frac{4 z_{j} X_{n}^{(2)}\left(z_{j}\right)}{z_{j}^{2}-1}
\end{array}\right| \\
& =\left|h_{n}\right|^{2} \frac{\left|z_{j}-\alpha_{n}\right|^{2}}{\left(1-\left|\alpha_{n}\right|^{2}\right)\left|z_{j}^{2}-1\right|^{4}} \\
& \times\left\{2 \Re\left[z_{j} \overline{X_{n}^{(2)}\left(z_{j}\right)} X_{n}^{(2)^{\prime}}\left(z_{j}\right)\right]-\left|X_{n}^{(2)}\left(z_{j}\right)\right|^{2}\left(Q_{n+1}\left(z_{j}\right)+3\right)\right\} .
\end{aligned}
$$

From (17) we deduce that

$$
\begin{aligned}
X_{n}^{(2)^{\prime}}(z)=\frac{z^{3}\left(f_{n}+g_{n} z+z^{2}\right) B_{n-1}(z)}{1}-\overline{\overline{\alpha_{n}} z} \\
\quad \times\left[\frac{3}{z}+\frac{B_{n-1}^{\prime}(z)}{B_{n-1}(z)}+\frac{g_{n}+2 z+e_{n} \frac{1-\overline{\alpha_{n}} z}{z^{3} B_{n-1}(z)}}{f_{n}+g_{n} z+z^{2}}+\frac{\overline{\alpha_{n}}}{1-\overline{\alpha_{n}} z}\right],
\end{aligned}
$$

so that for $z \in \mathbb{T}$ it holds that

$$
\begin{aligned}
z X_{n}^{(2)^{\prime}}(z)= & {\left[X_{n}^{(2)}(z)-\left(d_{n}+e_{n} z\right)\right] } \\
& \times\left\{2+Q_{n}(z)+\frac{z\left(g_{n}+2 z+e_{n} \frac{1-\overline{z_{n}} z}{\bar{\alpha}_{n-1}(z)}\right)}{f_{n}+g_{n} z+z^{2}}+\frac{\overline{\alpha_{n}} z}{1-\overline{\alpha_{n}} z}\right\} \\
= & {\left[X_{n}^{(2)}(z)-\left(d_{n}+e_{n} z\right)\right] } \\
& \times\left\{3+Q_{n+1}(z)+\frac{z^{2} G_{n}(z)}{\left(f_{n}+g_{n} z+z^{2}\right)\left(z-\alpha_{n}\right)}\right\},
\end{aligned}
$$

where

$$
G_{n}(z)=\alpha_{n} f_{n} \bar{z}^{2}-2 f_{n} \bar{z}-\left(\alpha_{n}+g_{n}\right)+e_{n}\left|z-\alpha_{n}\right|^{2} \overline{z^{3} B_{n-1}(z)} .
$$


Consequently,

$$
\begin{aligned}
& \lambda_{j}^{-1}=\left|h_{n}\right|^{2} \frac{\left|z_{j}-\alpha_{n}\right|^{2}}{\left(1-\left|\alpha_{n}\right|^{2}\right)\left|z_{j}^{2}-1\right|^{4}} \\
& \times {\left[\left(\left|X_{n}^{(2)}\left(z_{j}\right)\right|^{2}-2 \Re\left\{\left(d_{n}+e_{n} z_{j}\right) \overline{X_{n}^{(2)}\left(z_{j}\right)}\right\}\right)\left[3+Q_{n+1}\left(z_{j}\right)\right]\right.} \\
&\left.+2 \Re\left\{\frac{z_{j}^{2} G_{n}\left(z_{j}\right)\left(\left|X_{n}^{(2)}\left(z_{j}\right)\right|^{2}-\left(d_{n}+e_{n} z_{j}\right) \overline{X_{n}^{(2)}\left(z_{j}\right)}\right)}{\left(f_{n}+g_{n} z_{j}+z_{j}^{2}\right)\left(z_{j}-\alpha_{n}\right)}\right\}\right] .
\end{aligned}
$$

Finally, we have that

$$
\begin{aligned}
&\left|X_{n}^{(2)}(z)\right|^{2}- 2 \Re\left\{\left(d_{n}+e_{n} z\right) \overline{X_{n}^{(2)}(z)}\right\}=\left|\frac{f_{n}+g_{n} z+z^{2}}{z-\alpha_{n}}\right|^{2}-\left|d_{n}+e_{n} z\right|^{2}, \\
&\left|X_{n}^{(2)}(z)\right|^{2}-\left(d_{n}+e_{n} z\right) \overline{X_{n}^{(2)}(z)} \\
&=\left|\frac{f_{n}+g_{n} z+z^{2}}{z-\alpha_{n}}\right|^{2}+\overline{d_{n}+e_{n} z} \frac{z^{3}\left(f_{n}+g_{n} z+z^{2}\right) B_{n-1}(z)}{1-\overline{\alpha_{n}} z}
\end{aligned}
$$

and 5

$$
z_{j}^{3} B_{n-1}\left(z_{j}\right)=-\overline{\tau_{n}} \frac{p_{2}^{(2)}\left(z_{j}\right)}{p_{2}^{(2) *}\left(z_{j}\right)},
$$

where $p_{2}^{(2)}(z)$ is given by (36). Consequently,

$$
G_{n}\left(z_{j}\right)=\alpha_{n} f_{n} \bar{z}_{j}^{2}-2 f_{n} \overline{z_{j}}-\left(\alpha_{n}+g_{n}\right)-\tau_{n} e_{n}\left|z_{j}-\alpha_{n}\right|^{2} \frac{p_{2}^{(2) *}\left(z_{j}\right)}{p_{2}^{(2)}\left(z_{j}\right)},
$$

so that

$$
\begin{aligned}
\lambda_{j}^{-1}= & \frac{\left|h_{n}\right|^{2}}{\left(1-\left|\alpha_{n}\right|^{2}\right)\left|z_{j}^{2}-1\right|^{4}} \\
\times & {\left[\left(\left|f_{n}+g_{n} z_{j}+z_{j}^{2}\right|^{2}-\left|d_{n}+e_{n} z_{j}\right|^{2}\left|z_{j}-\alpha_{n}\right|^{2}\right)\left[3+Q_{n+1}\left(z_{j}\right)\right]\right.} \\
& \left.\quad+2 \Re\left\{G_{n}\left(z_{j}\right)\left[\frac{\overline{f_{n}} z_{j}^{2}+\overline{g_{n}} z_{j}+1}{z_{j}-\alpha_{n}}-\overline{\tau_{n}}\left(\overline{d_{n}} z_{j}+\overline{e_{n}}\right) \frac{p_{2}^{(2)}\left(z_{j}\right)}{p_{2}^{(2) *}\left(z_{j}\right)}\right]\right\}\right] .
\end{aligned}
$$

Whenever $z_{j}=\epsilon$ for a certain $j \in\{1, \ldots, n\}$, computing $\lambda_{j}$ by the aid of (21) again requires two times the application of l'Hôpital's rule to compute $\overline{\chi_{n}^{(2)}(\epsilon)}$, $\overline{\chi_{n}^{(2) *}(\epsilon)},\left(\chi_{n}^{(2) *}\right)^{\prime}(\epsilon)$ and $\chi_{n}^{(2)^{\prime}}(\epsilon)$. This gives rise to tedious calculations along with an inappropriate expression for computational purposes. Since $z_{j} \neq z_{k}$ for $j \neq k$,

\footnotetext{
${ }^{5}$ If there are no $\tau_{n} \in \mathbb{T}$ and $\gamma \in \mathbb{T} \backslash\{ \pm 1\}$ so that $p_{2}^{(2) *}(\gamma)=0$, then the right-hand side of (38) is well defined. At this moment of writing no proof has been found for this statement in general, but a proof is given in the Appendix for the special case in which $n=1$ or $\alpha_{n}=0$.
} 
there can be at most one index $j$ for which $z_{j}=\epsilon$. Therefore, if $z_{k} \neq-\epsilon$ for every $k \in\{1, \ldots, n\} \backslash\{j\}, \lambda_{j}$ can be computed as follows:

$$
\lambda_{j}=\int_{-\pi}^{\pi} \omega_{2}(\theta) d \theta-\sum_{\substack{k=1 \\ k \neq j}}^{n} \lambda_{k}=\pi-\sum_{\substack{k=1 \\ k \neq j}}^{n} \lambda_{k} .
$$

If, on the other hand, there exist indices $j$ and $k$ in $\{1, \ldots, n\}$ so that $z_{j}=-z_{k}=1$, then $\lambda_{j}$ and $\lambda_{k}$ can be computed by solving the following system of equations:

$$
\begin{cases}\lambda_{j}+\lambda_{k} & =\pi-\sum_{\substack{s=1 \\ s \notin\{j, k\}}}^{n} \lambda_{s}, \\ \lambda_{j}\left|\chi_{1}^{(2)}(1)\right|^{2}+\lambda_{k}\left|\chi_{1}^{(2)}(-1)\right|^{2} & =1-\sum_{\substack{s=1 \\ s \notin\{j, k\}}}^{n} \lambda_{s}\left|\chi_{1}^{(2)}\left(z_{s}\right)\right|^{2},\end{cases}
$$

where we computed $\chi_{1}^{(2)}(z)$ with the aid of Maple 10 to find that

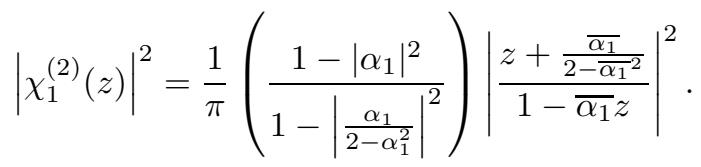

Note that there exists an index $j$ so that $z_{j}=\epsilon$ iff $\tau_{n}=-\frac{\phi_{n}^{(2)}(\epsilon)}{\phi_{n}^{(2) *}(\epsilon)}$. From Theorem 3.2 and proceeding as in (33) it follows that

$$
-\frac{\phi_{n}^{(2)}(\epsilon)}{\phi_{n}^{(2) *}(\epsilon)}=\frac{-1}{B_{n}(\epsilon)} \frac{X_{n}^{(2)^{\prime \prime}}(\epsilon)}{\overline{X_{n}^{(2))^{\prime \prime}}(\epsilon)}} .
$$

Let

$$
Y_{n}^{(2)}(z)=3+Q_{n+1}(z)+\frac{z^{2} G_{n}(z)}{\left(f_{n}+g_{n} z+z^{2}\right)\left(z-\alpha_{n}\right)} .
$$

Then it follows from (37) that

$z X_{n}^{(2)^{\prime \prime}}(z)=X_{n}^{(2)^{\prime}}(z)\left(Y_{n}^{(2)}(z)-1\right)-e_{n} Y_{n}^{(2)}(z)+\left(X_{n}^{(2)}(z)-\left(d_{n}+e_{n} z\right)\right) Y_{n}^{(2)^{\prime}}(z)$.

It also follows from (37) that

$$
0=\epsilon X_{n}^{(2)^{\prime}}(\epsilon)=\left(X_{n}^{(2)}(\epsilon)-\left(d_{n}+e_{n} \epsilon\right)\right) Y_{n}^{(2)}(\epsilon)=-\left(d_{n}+e_{n} \epsilon\right) Y_{n}^{(2)}(\epsilon),
$$

so that $Y_{n}^{(2)}(\epsilon)=0$ due to Lemma 3.4. Thus,

$$
\begin{aligned}
X_{n}^{(2)^{\prime \prime}}(\epsilon) & =-\left(d_{n}+e_{n} \epsilon\right) \epsilon Y_{n}^{(2)^{\prime}}(\epsilon) \\
& =\left(d_{n}+e_{n} \epsilon\right)\left[\frac{\epsilon\left(k_{n}(\epsilon)+e_{n} \overline{B_{n-1}(\epsilon)} l_{n}(\epsilon)\right)}{\left(1+f_{n}+g_{n} \epsilon\right)^{2}\left(\epsilon-\alpha_{n}\right)^{2}}-\epsilon Q_{n+1}^{\prime}(\epsilon)\right] \\
& =R_{n, \epsilon}^{(2)} \epsilon^{\mathbf{i} \gamma_{n, \epsilon}^{(2)}}, \quad \epsilon \in\{ \pm 1\}, R_{n, \epsilon}^{(2)}>0 \text { and } \gamma_{n, \epsilon}^{(2)} \in[0,2 \pi),
\end{aligned}
$$

where

$$
\begin{aligned}
k_{n}(\epsilon)= & f_{n} \alpha_{n}\left(6-f_{n}\right)-\left(1+g_{n} \alpha_{n}\right)\left(\alpha_{n}+g_{n}\right)-f_{n}\left(g_{n}+4 \epsilon\right)\left(1+\alpha_{n}^{2}\right) \\
l_{n}(\epsilon)= & \left|\epsilon-\alpha_{n}\right|^{2}\left[2\left(g_{n}-\alpha_{n}\right)+\epsilon\left(3+f_{n}-g_{n} \alpha_{n}\right)\right] \\
& \quad+\left[\left|\epsilon-\alpha_{n}\right|^{2} Q_{n}(\epsilon)-\epsilon 2 \mathbf{i} \Im\left\{\alpha_{n}\right\}\right]\left(1+f_{n}+g_{n} \epsilon\right)\left(\epsilon-\alpha_{n}\right),
\end{aligned}
$$


and with $Q_{n}(z)$ and $z Q_{n+1}^{\prime}(z)$ given, respectively, by (8) and (34). Consequently, there exists an index $j$ so that $z_{j}=\epsilon$ iff $\tau_{n}=\frac{e^{\mathrm{i}\left(2 \gamma_{n, \epsilon}^{(2)}-\pi\right)}}{B_{n}(\epsilon)}$.

Finally we have proved the following theorem.

Theorem 4.4. Let $\tau_{n} \in \mathbb{T}$ for $n \geq 1$ and suppose $\epsilon \in\{ \pm 1\}$. An n-point rational Szegö quadrature $I_{n}(f)$ for $I_{\omega_{2}}(f)$, given by (5) and (6), is then characterized as follows:

(1) Denote by $\left\{1,1,-1,-1, z_{1}, \ldots, z_{n}\right\}$ the set of zeros of $V_{n}^{(2)}\left(z, \tau_{n}\right)$, given by (35) and (36). Then $\left\{z_{1}, \ldots, z_{n}\right\}$ is the set of nodes for $I_{n}(f)$.

(2) For every $j \in\{1, \ldots, n\}$ for which $z_{j} \neq \epsilon$, the $j$ th weight is given by (39). Whenever $z_{j}=\epsilon$ for a certain $j \in\{1, \ldots, n\}$ and $z_{k} \neq-\epsilon$ for every $k \in\{1, \ldots, n\} \backslash\{j\}$, the $j$ th weight is given by (40). Finally, if there exist two distinct indices $j$ and $k$ in $\{1, \ldots, n\}$ so that $z_{j}=-z_{k}=1$, the associated weights are given by (41).

\section{SZEGŐ QUADRATURES ASSOCIATED WITH RATIONAL MODIFICATIONS of Chebyshev Weight FunCtions}

In this section we will consider a rational modification of a measure $\mu$ that is a measure of the form

$$
d \tilde{\mu}(\theta)=\frac{d \mu(\theta)}{|h(z)|^{2}} ; \quad z=e^{\mathbf{i} \theta},
$$

with $h(z)$ a given polynomial of degree $m$ whose zeros cannot be on $\mathbb{T}$. Without loss of generality, we can assume

$$
h(z)=\prod_{l=1}^{m}\left(z-\alpha_{l}\right), \quad\left|\alpha_{l}\right|<1, \alpha_{l} \neq 0, \quad l=1, \ldots, m .
$$

We will then consider Szegó quadrature formulas with respect to the measure $\tilde{\mu}$. In this respect, we recall that an $n$-point Szegö rule (2) for a measure $\mu$ has maximal domain of validity $\Lambda_{-(n-1), n-1}=\operatorname{span}\left\{z^{-(n-1)}, \ldots, z^{n-1}\right\}$, with dimension $2 n-1$, and that the nodes are the zeros of a para-orthogonal polynomial associated with $\mu$; see e.g. 11, 8, 9, 10, 11, 22, 24, 25. We also recall that for $\kappa_{n} \in \mathbb{T}, \kappa_{n}$-invariant para-orthogonal polynomials associated with $\mu$ are characterized in [25] as

$$
\Phi_{n}(z)=C_{n}\left[\rho_{n}(z)+\tau_{n} \rho_{n}^{*}(z)\right] \in \mathbb{P}_{n}, n \geq 1, C_{n} \in \mathbb{C} \backslash\{0\},
$$

where $\tau_{n}=\frac{\overline{C_{n} \kappa_{n}}}{C_{n}} \in \mathbb{T}$ and $\left\{\rho_{k}(z)\right\}_{k=0}^{\infty}$ is a sequence of Szegö polynomials associated with $\mu$; see e.g. [26], [28].

When $d \mu(\theta)=d \theta$, rational modifications of the Lebesgue measure appear such that Szegö polynomials for $\tilde{\mu}$ were earlier considered by Szegö in [28]. Observe that when $m=1, \tilde{\mu}$ gives rise to the so-called Poisson kernel. In relation to this, Waadeland considered in 33 for the first time Szegö quadrature formulas for the measure $d \tilde{\mu}(\theta)=\frac{d \theta}{|z-\alpha|^{2}}$, providing one of the first examples of this type of quadratures in the literature.

Rational modifications of the Lebesgue measure are very suitable in order to approximate other measures. For instance, the following important result is given in [26, Theorem 1.7.8]: Let $\eta$ be a probability measure supported on $\mathbb{T}$, and suppose 
$\left\{\varphi_{n}(z)\right\}_{n=0}^{\infty}$ is a sequence of orthonormal polynomials associated with $\eta$. For every $n$ it then holds that

$$
\int_{-\pi}^{\pi} \frac{d \theta}{2 \pi\left|\varphi_{n}\left(e^{\mathbf{i} \theta}\right)\right|^{2}}=1
$$

and

$$
d \eta_{n}(\theta)=\frac{d \theta}{2 \pi\left|\varphi_{n}\left(e^{\mathbf{i} \theta}\right)\right|^{2}} \longrightarrow d \eta \text { weakly when } n \rightarrow \infty .
$$

Consider now the polynomial $h(z)$ given by (44) and set $\alpha_{k}=0$ for every $k>m$. For every $n \geq m$ we then have that

$$
\pi_{n}^{*}(z)=\prod_{j=1}^{n}\left(z-\alpha_{j}\right)=z^{n-m} h(z), \quad \pi_{n}(z)=\prod_{j=1}^{n}\left(1-\overline{\alpha_{j}} z\right)=\prod_{j=1}^{m}\left(1-\overline{\alpha_{j}} z\right)=h^{*}(z) .
$$

So, let $\chi_{n}(z)$ denote the $n$th orthonormal rational function associated with $\mu$. Then for every $n \geq m, \chi_{n}(z)=\frac{P_{n}(z)}{h^{*}(z)}$ with $P_{n}(z) \in \mathbb{P}_{n} \backslash \mathbb{P}_{n-1}$. Moreover, if $n>m$, we have that $\left\langle\chi_{n}(z), u_{k}(z)\right\rangle_{\mu}=0$, where $u_{k}(z)=\frac{z^{k}}{h^{*}(z)}$ with $k=0,1, \ldots, n-1$, implying that

$$
0=\int_{-\pi}^{\pi} \frac{P_{n}(z)}{h^{*}(z)} \overline{\left(\frac{z^{k}}{h^{*}(z)}\right)} d \mu(\theta)=\int_{-\pi}^{\pi} P_{n}(z) \overline{z^{k}} \frac{d \mu(\theta)}{\left|h^{*}(z)\right|^{2}}=\int_{-\pi}^{\pi} P_{n}(z) \overline{z^{k}} \frac{d \mu(\theta)}{|h(z)|^{2}}
$$

with $z=e^{i \theta}$ and $k=0,1, \ldots, n-1$. Consequently, the numerator of the $n$th orthonormal rational function associated with the measure $\mu$ coincides for $n>m$ with an $n$th orthonormal polynomial associated with the measure $\tilde{\mu}$ given by (43).

Example 5.1. Take the Lebesgue measure $d \mu(\theta)=\omega_{1}(\theta) d \theta \equiv d \theta$; see (6). Then for every $n>m$ it follows from (7) that

$$
\begin{aligned}
\chi_{n}(z) & =c_{n} z \prod_{j=1}^{n-1}\left(\frac{z-\alpha_{j}}{1-\overline{\alpha_{j}} z}\right)=c_{n} z \prod_{j=1}^{m}\left(\frac{z-\alpha_{j}}{1-\overline{\alpha_{j}} z}\right) \prod_{j=m+1}^{n-1}\left(\frac{z-\alpha_{j}}{1-\overline{\alpha_{j}} z}\right) \\
& =c_{n} \frac{z^{n-m} h(z)}{h^{*}(z)}, \quad c_{n} \neq 0 .
\end{aligned}
$$

Hence, $P_{n}(z)=z^{n-m} h(z)$ represents the $n$th monic Szegő polynomial for the measure $d \tilde{\mu}(\theta)=\frac{d \theta}{|h(z)|^{2}}$ with $z=e^{\mathbf{i} \theta}$ (compare with the first approach given in [28, pp. 289-290]; actually, this expression for $P_{n}(z)$ also holds for $\left.n=m\right)$.

Let us see next what happens with the quadrature formulas. Assume as above $\alpha_{k}=0$ for $k>m$ and $\alpha_{l} \in \mathbb{D} \backslash\{0\}$ for $l=1, \ldots, m$. Let $h(z)=\prod_{l=1}^{m}\left(z-\alpha_{l}\right) \in$ $\mathbb{P}_{m} \backslash \mathbb{P}_{m-1}$, and suppose $n>m$. From Theorem 2.2 we then have that

$$
I_{n}(f)=\sum_{j=1}^{n} \lambda_{j} f\left(z_{j}\right)=I_{\mu}(f)=\int_{-\pi}^{\pi} f\left(e^{\mathbf{i} \theta}\right) d \mu(\theta), \quad \forall f \in \mathcal{R}_{n-1} .
$$

Consider now the case in which $f(z)=\frac{z^{k}}{\pi_{n-1}^{*}(z) \pi_{n-1}(z)} \in \mathcal{R}_{n-1}$ for $k=0,1, \ldots, 2 n-$ 2 , and observe that $\pi_{n-1}(z)=z^{n-1} \overline{\pi_{n-1}^{*}(z)}$ for $z \in \mathbb{T}$. Taking into account that $k=0,1, \ldots, 2 n-2$, it then follows that

$$
f(z)=\frac{z^{k-(n-1)}}{\left|\pi_{n-1}^{*}(z)\right|^{2}}=\frac{z^{k+1-n}}{|h(z)|^{2}}=\frac{z^{s}}{|h(z)|^{2}}, \quad-(n-1) \leq s \leq n-1, \quad z \in \mathbb{T} .
$$


Hence,

$$
\begin{aligned}
\int_{-\pi}^{\pi} f(z) d \mu(\theta) & =\int_{-\pi}^{\pi} \frac{z^{s}}{|h(z)|^{2}} d \mu(\theta)=\sum_{j=1}^{n} \lambda_{j} \frac{z_{j}^{s}}{\left|h\left(z_{j}\right)\right|^{2}}=\sum_{j=1}^{n} \tilde{\lambda}_{j} z_{j}^{s} \\
& =\int_{-\pi}^{\pi} z^{s} d \tilde{\mu}(\theta), \quad z=e^{i \theta},-(n-1) \leq s \leq n-1,
\end{aligned}
$$

where $\tilde{\lambda}_{j}=\frac{\lambda_{j}}{\left|h\left(z_{j}\right)\right|^{2}}$ for $j=1, \ldots, n$ and $d \tilde{\mu}(\theta)$ is given by (43). It follows from Theorem 2.2 that $\left\{z_{j}\right\}_{j=1}^{n}$ are the zeros of $\Psi_{n}\left(z, \tau_{n}\right)=\chi_{n}(z)+\tau_{n} \chi_{n}^{*}(z)$. Furthermore, setting $\chi_{n}(z)=\frac{P_{n}(z)}{\pi_{n}(z)}$ and

$$
\chi_{n}^{*}(z)=B_{n}(z) \chi_{n *}(z)=B_{n}(z) \frac{\overline{P_{n}(1 / \bar{z})}}{\overline{\pi_{n}(1 / \bar{z})}}=\frac{B_{n}(z) P_{n}^{*}(z)}{z^{n} \overline{\pi_{n}(1 / \bar{z})}}=\frac{P_{n}^{*}(z)}{\pi_{n}(z)},
$$

we obtain that

$$
\Psi_{n}\left(z, \tau_{n}\right)=\frac{P_{n}(z)+\tau_{n} P_{n}^{*}(z)}{\pi_{n}(z)}=\frac{P_{n}(z)+\tau_{n} P_{n}^{*}(z)}{h^{*}(z)} .
$$

Thus, we have proved the following theorem.

Theorem 5.2. Let $h(z)=\prod_{l=1}^{m}\left(z-\alpha_{l}\right)$ with $\alpha_{l} \in \mathbb{D} \backslash\{0\}$ for every $l=1, \ldots, m$, and set $\alpha_{k}=0$ for every $k>m$. Further, let $n>m$ and suppose $\chi_{n}(z)=\frac{P_{n}(z)}{h^{*}(z)}$ with $P_{n}(z) \in \mathbb{P}_{n} \backslash \mathbb{P}_{n-1}$ is the $n$th orthonormal rational function associated with the measure $\mu$. Then for a given $\tau_{n} \in \mathbb{T}, I_{n}(f)=\sum_{j=1}^{n} \tilde{\lambda}_{j} f\left(z_{j}\right)$ is an n-point Szegö quadrature formula for $d \tilde{\mu}(\theta)=\frac{d \mu(\theta)}{\left|h\left(e^{i \theta}\right)\right|^{2}}$, if and only if

(1) the nodes $\left\{z_{j}\right\}_{j=1}^{n}$ are the zeros of $P_{n}(z)+\tau_{n} P_{n}^{*}(z)$, and

(2) $\tilde{\lambda}_{j}=\frac{\lambda_{j}}{\left|h\left(z_{j}\right)\right|^{2}}$, where $\left\{\lambda_{j}\right\}_{j=1}^{n}$ is the set of weights corresponding to the $n$ point rational Szegö quadrature for $\mu$.

As an application of the previous theorem, we will now deduce characterization theorems for Szegö quadrature formulas associated with the measures $d \tilde{\mu}(\theta)=$ $\frac{\omega_{i}(\theta) d \theta}{|h(z)|^{2}}$, where $z=e^{\mathbf{i} \theta}$ and $\omega_{i}(\theta)$ is given by (6) for $i \in\{1, \ldots, 4\}$.

We start with the Lebesgue measure (compare with [12, Theorem 4.2]).

Theorem 5.3. Assume $h(z)=\prod_{l=1}^{m}\left(z-\alpha_{l}\right)$ with $\alpha_{l} \in \mathbb{D} \backslash\{0\}$ for every $l=$ $1, \ldots, m$. Let $\tau_{n} \in \mathbb{T}$ and set $d \tilde{\mu}(\theta)=\frac{d \theta}{\left|h\left(e^{i \theta}\right)\right|^{2}}$. An n-point Szegö formula for $\tilde{\mu}$ and $n>m$ is then given by

$$
I_{n}(f)=\sum_{j=1}^{n} \tilde{\lambda}_{j} f\left(z_{j}\right),
$$

where the nodes $\left\{z_{j}\right\}_{j=1}^{n}$ are the zeros of $z^{n-m} h(z)+\tau_{n} h^{*}(z)$ and the weights $\left\{\tilde{\lambda}_{j}\right\}_{j=1}^{n}$ are given by

$$
\tilde{\lambda}_{j}=\frac{2 \pi}{\left|h\left(z_{j}\right)\right|^{2}\left[n-m+\sum_{l=1}^{m} \frac{1-\left|\alpha_{l}\right|^{2}}{\left|z_{j}-\alpha_{l}\right|^{2}}\right]} ; j=1, \ldots, n .
$$

In the following theorem we characterize a Szegő quadrature formula associated with a rational modification of the Chebyshev weight functions $1 \pm \cos \theta$.

Theorem 5.4. Suppose $h(z)=\prod_{l=1}^{m}\left(z-\alpha_{l}\right)$ with $\left\{\alpha_{l}\right\}_{l=1}^{m} \subset \mathbb{D} \backslash\{0\}, n>m$, $\tau_{n} \in \mathbb{T}, \nu_{i}=(-1)^{i-1}$ for fixed $i \in\{3,4\}$, and let

$$
d \tilde{\mu}(\theta)=\frac{1-\nu_{i} \cos \theta}{\left|h\left(e^{\mathbf{i} \theta}\right)\right|^{2}} d \theta .
$$


Next, let $Q_{n}(z)=n-m+\sum_{l=1}^{m} \frac{1-\left|\alpha_{l}\right|^{2}}{\left|z-\alpha_{l}\right|^{2}}$ and define $\tilde{Q}_{n}^{(i)}=\left[1+Q_{n}\left(\nu_{i}\right)\right]^{-1}$. Further, assume $a_{n}^{(i)}=\nu_{i}^{n} \frac{h\left(\nu_{i}\right)}{h\left(\nu_{i}\right)} \tilde{Q}_{n}^{(i)}$ and $b_{n}^{(i)}=\nu_{i}\left(1+\tilde{Q}_{n}^{(i)}\right)$. An n-point Szegö formula for $\tilde{\mu}$ is then given by $I_{n}(f)=\sum_{j=1}^{n} \tilde{\lambda}_{j} f\left(z_{j}\right)$, where the set of nodes and weights are determined as follows:

(1) Denote by $\left\{\nu_{i}, \nu_{i}, z_{1}, \ldots, z_{n}\right\}$ the set of zeros of

$$
\begin{aligned}
V_{n}^{(i)}\left(z, \tau_{n}\right)= & z^{n+1-m}\left[-b_{n}^{(i)}+\left(1+\tau_{n} \overline{a_{n}^{(i)}}\right) z\right] h(z) \\
& +z^{m}\left[a_{n}^{(i)}+\tau_{n}\left(1-b_{n}^{(i)} z\right)\right] \overline{h(z)} .
\end{aligned}
$$

Then $\left\{z_{1}, \ldots, z_{n}\right\}$ is the set of nodes for $I_{n}(f)$.

(2) For every $j \in\{1, \ldots, n\}$ for which $z_{j} \neq \nu_{i}$, the $j$ th weight is given by

$$
\begin{aligned}
& \tilde{\lambda}_{j}^{-1}=\nu_{i} \frac{\left|h\left(z_{j}\right)\right|^{2}\left(\left|z_{j}-b_{n}^{(i)}\right|^{2}-\left|a_{n}^{(i)}\right|^{2}\right)}{\pi b_{n}^{(i)}\left|z_{j}-\nu_{i}\right|^{4}} \\
& \times\left[1+Q_{n+1}\left(z_{j}\right)+2 b_{n}^{(i)} \Re\left\{\left[-b_{n}^{(i)}+\left(1+\tau_{n} \overline{a_{n}^{(i)}}\right) z_{j}\right]^{-1}\right\}\right] .
\end{aligned}
$$

Whenever $z_{j}=\nu_{i}$ for a certain $j \in\{1, \ldots, n\}$, the associated weight is given by $\tilde{\lambda}_{j}=\lambda_{j} /\left|h\left(z_{j}\right)\right|^{2}$, where $\lambda_{j}$ is given by (32).

Finally, for the remainder Chebyshev weight function we have the following theorem.

Theorem 5.5. Suppose $h(z)=\prod_{l=1}^{m}\left(z-\alpha_{l}\right)$ with $\left\{\alpha_{l}\right\}_{l=1}^{m} \subset \mathbb{D} \backslash\{0\}, n>m$, $\tau_{n} \in \mathbb{T}, \epsilon \in\{ \pm 1\}$, and let

$$
d \tilde{\mu}(\theta)=\frac{\sin ^{2} \theta}{\left|h\left(e^{\mathbf{i} \theta}\right)\right|^{2}} d \theta .
$$

Next, let $Q_{n}(z)=n-m+\sum_{l=1}^{m} \frac{1-\left|\alpha_{l}\right|^{2}}{\left|z-\alpha_{l}\right|^{2}}$ and define

$$
a^{+}=\frac{h(1)}{\overline{h(1)}}, \quad a^{-}=(-1)^{n-1} \frac{h(-1)}{\overline{h(-1)}}, \quad b^{+}=2+Q_{n}(1), \quad b^{-}=2+Q_{n}(-1) .
$$

Further, assume $\underline{x}=\left(d_{n}, e_{n}, f_{n}, g_{n}\right)^{T}$ is the solution of the system given by (15) and (16). An n-point Szegö formula for $\tilde{\mu}$ is then given by $I_{n}(f)=\sum_{j=1}^{n} \tilde{\lambda}_{j} f\left(z_{j}\right)$, where the set of nodes and weights are determined as follows:

(1) Let $\left\{+1+1,-1,-1, z_{1}, \ldots, z_{n}\right\}$ be the set of zeros of

$$
\begin{aligned}
V_{n}^{(2)}\left(z, \tau_{n}\right)= & z^{n+2-m}\left[\left(1+\tau_{n} \overline{d_{n}}\right) z^{2}+\left(g_{n}+\tau_{n} \overline{e_{n}}\right) z+f_{n}\right] h(z) \\
& +z^{m}\left[\tau_{n} \overline{f_{n}} z^{2}+\left(e_{n}+\tau_{n} \overline{g_{n}}\right) z+\tau_{n}+d_{n}\right] \overline{h(z)} .
\end{aligned}
$$

Then $\left\{z_{1}, \ldots, z_{n}\right\}$ is the set of nodes of $I_{n}(f)$.

(2) For every $j \in\{1, \ldots, n\}$ for which $z_{j} \neq \epsilon$, the $j$ th weight is given by

$$
\begin{aligned}
\tilde{\lambda}_{j}^{-1}= & \frac{2\left|h\left(z_{j}\right)\right|^{2}\left(\left|d_{n}+e_{n} z_{j}\right|^{2}-\left|f_{n}+g_{n} z_{j}+z_{j}^{2}\right|^{2}\right)}{\pi f_{n}\left|z_{j}^{2}-1\right|^{4}} \\
& \times\left[3+Q_{n+1}\left(z_{j}\right)-\frac{2 \Re\left\{\overline{\tau_{n}}\left(2 f_{n} \overline{z_{j}}+g_{n}\right) \overline{z_{j}} p_{2}^{(2)}\left(z_{j}\right)+e_{n} z_{j} \overline{p_{2}^{(2)}\left(z_{j}\right)}\right\}}{\left|p_{2}^{(2)}\left(z_{j}\right)\right|^{2}}\right],
\end{aligned}
$$


where $p_{2}^{(2)}\left(z_{j}\right)=\left(d_{n}+e_{n} z_{j}\right)+\tau_{n}\left(\overline{f_{n}} z_{j}^{2}+\overline{g_{n}} z_{j}+1\right)$. Whenever $z_{j}=\epsilon$ for a certain $j \in\{1, \ldots, n\}$ and $z_{k} \neq-\epsilon$ for every $k \in\{1, \ldots, n\} \backslash\{j\}$, the $j$ th weight is given by $\tilde{\lambda}_{j}=\lambda_{j} /\left|h\left(z_{j}\right)\right|^{2}$, where $\lambda_{j}$ is given by (40). Finally, if there exist two distinct indices $j$ and $k$ in $\{1, \ldots, n\}$ so that $z_{j}=-z_{k}=1$, the associated weights are given by $\tilde{\lambda}_{j}=\lambda_{j} /\left|h\left(z_{j}\right)\right|^{2}$ and $\tilde{\lambda}_{k}=\lambda_{k} /\left|h\left(z_{k}\right)\right|^{2}$, where $\lambda_{j}$ and $\lambda_{k}$ are given by (41) and (42).

\section{Numerical EXAmples}

The aim of this final section is to present some numerical illustrations of the results given in Section 5 in the construction of Szegö-type quadrature formulas with respect to rational modifications of Chebyshev weight functions.

We start by recalling that the parameter $\tau_{n} \in \mathbb{T}$ in (45) can be choosen freely to fix a complex number $\lambda \in \mathbb{T}$ as a node of the Szegö quadrature formula. By setting $\tau_{n}=-\frac{\rho_{n}(\lambda)}{\lambda^{n} \overline{\rho_{n}(\lambda)}} \in \mathbb{T}$, the so-called "Szegö-Radau rules" arise (see e.g. [10, Proposition 2.8]). Recently, Jagels and Reichel have characterized Szegö-Lobatto quadrature formulas in [24], i.e. Szegö rules with two prescribed nodes on $\mathbb{T}$. Suppose $z_{\alpha}$ and $z_{\beta}$ are two distinct points on $\mathbb{T}$. Let $N \geq 2$ be fixed, and assume there exist $N-2$ distinct nodes $z_{1}, \ldots, z_{N-2}$ on $\mathbb{T}$ such that $z_{j} \neq z_{\alpha}$ and $z_{j} \neq z_{\beta}$ for $1 \leq j \leq N-2$. Furthermore, suppose there exist positive weights $A_{1}, A_{2}, \lambda_{1}, \ldots, \lambda_{N-2}$ so that

$$
\begin{aligned}
& \forall f \in \Lambda_{-(N-2), N-2}: \\
& \qquad I_{N}(f):=A_{1} f\left(z_{\alpha}\right)+A_{2} f\left(z_{\beta}\right)+\sum_{j=1}^{N-2} \lambda_{j} f\left(z_{j}\right)=\int_{\mathbb{T}} f(z) d \mu(z)=: I_{\mu}(f) .
\end{aligned}
$$

Then, $I_{N}(f)$ is called an $N$-point Szegö-Lobatto quadrature formula for $\mu$ with prescribed nodes $z_{\alpha}$ and $z_{\beta}$. We now have the following theorem (for the proof, see 24]).

Theorem 6.1 (Szegő-Lobatto quadrature). Let $z_{\alpha}$ and $z_{\beta}$ be two distinct fixed points on $\mathbb{T}$ and set $a=z_{\alpha}^{n-1} \frac{\overline{\rho_{n}\left(z_{\alpha}\right)}}{\rho_{n}\left(z_{\alpha}\right)} \in \mathbb{T}$ and $b=z_{\beta}^{n-1} \frac{\overline{\rho_{n}\left(z_{\beta}\right)}}{\rho_{n}\left(z_{\beta}\right)} \in \mathbb{T}$. Then one of the following statements holds:

(1) If $a z_{\alpha}=b z_{\beta}$, we have that an n-point Szegö formula $I_{n}(f)$ has $z_{\alpha}$ and $z_{\beta}$ as nodes by setting $\tau_{n}=-\frac{\rho_{n}\left(z_{\alpha}\right)}{\rho_{n}^{*}\left(z_{\alpha}\right)}=-\frac{\rho_{n}\left(z_{\beta}\right)}{\rho_{n}^{*}\left(z_{\beta}\right)} \in \mathbb{T}$.

(2) If $a=b$, an $(n+1)$-point Szegö formula $I_{n+1}(f)$ has $z_{\alpha}$ and $z_{\beta}$ as nodes by setting $\tau_{n+1}=-z_{\alpha} \frac{\rho_{n}\left(z_{\alpha}\right)}{\rho_{n}^{*}\left(z_{\alpha}\right)}=-z_{\beta} \frac{\rho_{n}\left(z_{\beta}\right)}{\rho_{n}^{*}\left(z_{\beta}\right)} \in \mathbb{T}$.

(3) Suppose $a z_{\alpha} \neq b z_{\beta}$ and $a \neq b$, and denote by $\Gamma$ the circle with center $c=$ $-\frac{z_{\alpha}-z_{\beta}}{a z_{\alpha}-b z_{\beta}}$ and radius $r=\left|\frac{a-b}{a z_{\alpha}-b z_{\beta}}\right|$. Let $\tilde{\delta}_{n+1} \in \Gamma \cap \mathbb{D}$, a circular arc that is proved to be a non-empty set, and set $\tau_{n+2}=-\frac{a z_{\alpha}-b z_{\beta}}{a-b} \tilde{\delta}_{n+1}-\frac{z_{\alpha}-z_{\beta}}{a-b} \in$ $\mathbb{T}$. Then, there exists an $(n+2)$-point Szegö-Lobatto quadrature formula whose nodes are the zeros of $\tilde{\Phi}_{n+2}\left(z, \tau_{n+2}\right)=z \tilde{\rho}_{n+1}(z)+\tau_{n+2} \tilde{\rho}_{n+1}^{*}(z)$ with $\tilde{\rho}_{n+1}(z)=z \rho_{n}(z)+\tilde{\delta}_{n+1} \rho_{n}^{*}(z)$.

Remark 6.2. In the special case in which $\tilde{\delta}_{n+1}=\rho_{n+1}(0)$ in Theorem 6.1 (3), the $(n+2)$-point Szegö-Lobatto formula is actually an $(n+2)$-point Szegő formula and consequently, exact in $\Lambda_{-(n+1), n+1}$. 


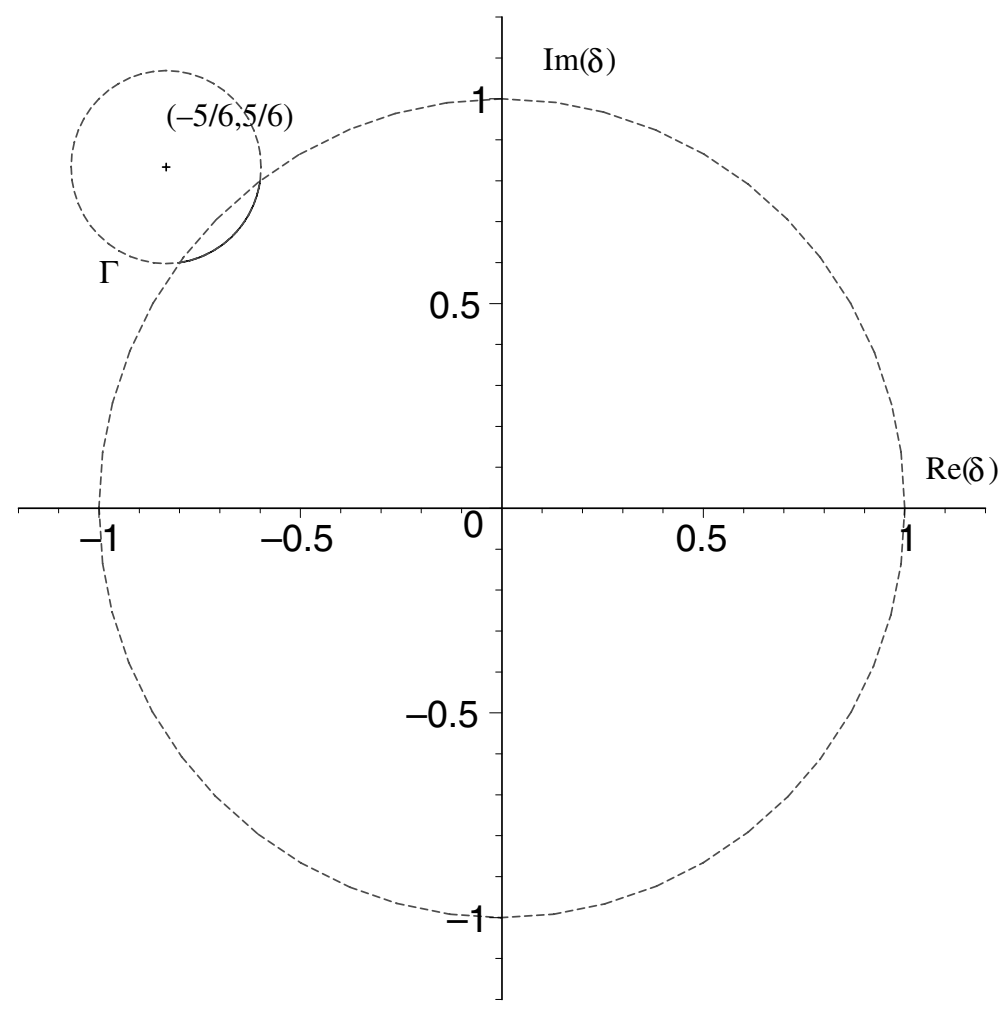

FiguRE 1. The circular arc $\Gamma \cap \mathbb{D}$.

In analogy with the real line situation (see e.g. [19]), the set of Szegö, SzegöRadau and Szegö-Lobatto rules we call Szegö-type quadratures.

Example 6.3. Consider the rational modification of the Lebesgue measure given by

$$
d \tilde{\mu}(\theta)=\frac{d \theta}{5 / 4-\sin (2 \theta)}=\frac{d \theta}{\left|z^{2}-\mathbf{i} / 2\right|^{2}}, \quad z=e^{\mathbf{i} \theta} .
$$

From Example 5.1 it then follows that the corresponding monic Szegó polynomials are explicitly given by $\rho_{n}(z)=z^{n-2}\left(z^{2}-\mathbf{i} / 2\right)$ for every $n \geq 2$. Next, let $n=10$, and suppose $z_{\alpha}=1$ and $z_{\beta}=\mathbf{i}$. From Theorem 6.1 we then deduce that

$$
a=\frac{3+4 \mathbf{i}}{5} \text { and } b=\frac{4+3 \mathbf{i}}{5}
$$

where clearly $a-b=\frac{-1+\mathbf{i}}{5} \neq 0$ and $a z_{\alpha}-b z_{\beta}=\frac{6}{5} \neq 0$. Consequently, we are dealing with the third situation of Theorem 6.1. Elementary calculation yields that $\Gamma$ is the circle with center $c=\frac{5}{6}\left(-1+\mathbf{i}\right.$ ) and radius $r=\frac{\sqrt{2}}{6}$, and $\tilde{\delta}_{11} \in \Gamma \cap \mathbb{D}$ iff (see also Figure 1)

$$
\tilde{\delta}_{11}=c+r e^{\mathbf{i} \theta}, \text { with }-\theta_{0}<\theta<-\theta_{1}, \theta_{0}=\arctan (7), \theta_{1}=\frac{\pi}{2}-\theta_{0} .
$$

Since $\rho_{11}(0)=0 \notin \Gamma \cap \mathbb{D}$, it follows that the 12-point Szegö-Lobatto formula associated with $\tilde{\delta}_{11}$ is not a 12-point Szegö formula (see Remark 6.2); thus, the 
domain of exactness is $\Lambda_{-10,10}$. Therefore, the nodes in the 12-point Szegö-Lobatto formula associated with $\tilde{\delta}_{11}$ are the zeros of $\tilde{\Phi}_{12}\left(z, \tau_{12}\right)$, given by

$$
\tilde{\Phi}_{12}\left(z, \tau_{12}\right)=z \tilde{\rho}_{11}(z)+\tau_{12} \tilde{\rho}_{11}^{*}(z)
$$

where

$$
\tilde{\rho}_{11}(z)=z \rho_{10}(z)+\tilde{\delta}_{11} \rho_{10}^{*}(z)=z^{9}\left(z^{2}-\mathbf{i} / 2\right)+\tilde{\delta}_{11}\left(1+z^{2} \mathbf{i} / 2\right),
$$

and $\tau_{12}=3(1+\mathbf{i}) \tilde{\delta}_{11}+5$. Let $z_{j}, j=1, \ldots, 12$, denote the zeros of $\tilde{\Phi}_{12}\left(z, \tau_{12}\right)$. We have used Maple 10 with 40 digits to compute these zeros for the case in which $\tilde{\delta}_{11}=-\frac{3}{4}+\frac{10-\sqrt{7}}{12} \mathbf{i}($ that is, (46)) with $\theta=-\arctan (\sqrt{7}))$ and $\tau_{12}=\frac{\sqrt{7}+1}{4}-\frac{\sqrt{7}-1}{4} \mathbf{i}$, respectively, for the case in which $\tilde{\delta}_{11}=\frac{2}{3}(-1+\mathbf{i}$ ) (that is, (46) with $\theta=-\pi / 4$ ) and $\tau_{12}=1$. The results are given in Table 1, respectively, Table 2

Finally, since $\tilde{\delta}_{11} \neq \rho_{11}(0)$, we are dealing with a modified measure so that we can not use Theorem $\left[5.3\right.$ to compute the corresponding weights. Let $\tilde{\varphi}_{11}(z)$ denote the polynomial of degree 11 that is orthonormal with respect to this modified measure. We then have the following Christoffel-Darboux formula in the ordinary polynomial situation (see also [5, Theorem 3.1.3]):

$$
\mathcal{K}_{11}(z, \xi)=\frac{\tilde{\varphi}_{11}^{*}(z) \overline{\tilde{\varphi}_{11}^{*}(\xi)}-z \bar{\xi} \tilde{\varphi}_{11}(z) \overline{\tilde{\varphi}_{11}(\xi)}}{1-z \bar{\xi}} .
$$

Setting $\lambda_{j}^{-1}=\mathcal{K}_{11}\left(z_{j}, z_{j}\right)$ and proceeding as in Section 4, it is easily verified that

$$
\lambda_{j}^{-1}=2 \Re\left\{z_{j} \overline{\tilde{\varphi}_{11}\left(z_{j}\right)} \tilde{\varphi}_{11}^{\prime}\left(z_{j}\right)\right\}-10\left|\tilde{\varphi}_{11}\left(z_{j}\right)\right|^{2} .
$$

Further, with $\tilde{\varphi}_{11}(z)=c \tilde{\rho}_{11}(z)$ we obtain that

$$
\lambda_{j}^{-1}=|c|^{2} \mu_{j}^{-1}, \quad \mu_{j}^{-1}:=2 \Re\left\{z_{j} \overline{\tilde{\rho}_{11}\left(z_{j}\right)} \tilde{\rho}_{11}^{\prime}\left(z_{j}\right)\right\}-10\left|\tilde{\rho}_{11}\left(z_{j}\right)\right|^{2},
$$

so that it remains to determine $|c|^{2}$. It should now hold that

$$
\sum_{j=1}^{12} \lambda_{j}=\int_{-\pi}^{\pi} \frac{d \theta}{\left|e^{2 \mathbf{i} \theta}-\mathbf{i} / 2\right|^{2}}=\frac{8 \pi}{3}=\frac{\sum_{j=1}^{12} \mu_{j}}{|c|^{2}},
$$

and hence,

$$
|c|^{2}=\frac{3}{8 \pi} \sum_{j=1}^{12} \mu_{j} .
$$

The resulting weights are given in Tables 1 and 2 for the case in which $\tilde{\delta}_{11}=-\frac{3}{4}+$ $\frac{10-\sqrt{7}}{12} \mathbf{i}$ and $\tau_{12}=\frac{\sqrt{7}+1}{4}-\frac{\sqrt{7}-1}{4} \mathbf{i}$, respectively, for the case in which $\tilde{\delta}_{11}=\frac{2}{3}(-1+\mathbf{i})$ and $\tau_{12}=1$.

With these nodes and weights, the integral is computed to within the numerical precision for

$$
\int_{-\pi}^{\pi}\left(\frac{5}{4}-\sin (2 \theta)\right) d \tilde{\mu}(\theta), \quad \text { and for } \quad \int_{-\pi}^{\pi} e^{\mathrm{i} k \theta} d \tilde{\mu}(\theta), k=-10, \ldots, 10 .
$$

Example 6.4. Consider the rational modifications

$$
d \tilde{\mu}(\theta)=\frac{\omega_{i}(\theta) d \theta}{|z-1 / 4|^{2}}, \quad i=2,3,4, \quad z=e^{\mathbf{i} \theta}
$$


TABLE 1. The nodes $z_{j}$ and weights $\lambda_{j}$ in the 12-point SzegóLobatto formula for the case in which $\tilde{\delta}_{11}=-\frac{3}{4}+\frac{10-\sqrt{7}}{12} \mathbf{i}$ and $\tau_{12}=\frac{\sqrt{7}+1}{4}-\frac{\sqrt{7}-1}{4} \mathbf{i}$.

\begin{tabular}{|c|c|c|}
\hline$j$ & $z_{j}$ & $\lambda_{j}$ \\
\hline 1 & 1 & 0.4516560678 \\
2 & $0.9638145989+0.2665734776 \mathbf{i}$ & 0.1232452240 \\
3 & $0.8316896358+0.5552408033 \mathbf{i}$ & 1.3564329045 \\
4 & $0.5255107494+0.8507869605 \mathbf{i}$ & 1.3244867113 \\
5 & $\mathbf{i}$ & 0.4907276839 \\
6 & $-0.5936593393+0.8047164649 \mathbf{i}$ & 0.2935974363 \\
7 & $-0.9584388804+0.2852979365 \mathbf{i}$ & 0.3549235398 \\
8 & $-0.9499460287-0.3124140564 \mathbf{i}$ & 0.8469595298 \\
9 & $-0.7027746634-0.7114125192 \mathbf{i}$ & 1.6733022550 \\
10 & $-0.2969879126-0.9548812386 \mathbf{i}$ & 0.8202977524 \\
11 & $0.3024297035-0.9531716920 \mathbf{i}$ & 0.3490662051 \\
12 & $0.8140918496-0.5807361367 \mathbf{i}$ & 0.2928850997 \\
\hline
\end{tabular}

TABle 2. The nodes $z_{j}$ and weights $\lambda_{j}$ in the 12-point SzegöLobatto formula for the case in which $\tilde{\delta}_{11}=\frac{2}{3}(-1+\mathbf{i})$ and $\tau_{12}=1$.

\begin{tabular}{|c|c|c|}
\hline$j$ & $z_{j}$ & $\lambda_{j}$ \\
\hline 1 & 1 & 0.4833219467 \\
2 & $0.8615961242+0.5075944433 \mathbf{i}$ & 1.1348377814 \\
3 & $(1+\mathbf{i}) / \sqrt{2}$ & 0.5132214093 \\
4 & $0.5075944433+0.8615961242 \mathbf{i}$ & 1.1348377814 \\
5 & $\mathbf{i}$ & 0.4833219467 \\
6 & $-0.5892211604+0.8079717966 \mathbf{i}$ & 0.2926150925 \\
7 & $-0.9562662716+0.2924975517 \mathbf{i}$ & 0.3518577037 \\
8 & $-0.9522800017-0.3052258155 \mathbf{i}$ & 0.8334298010 \\
9 & $-(1+\mathbf{i}) / \sqrt{2}$ & 1.6722343497 \\
10 & $-0.3052258155-0.9522800017 \mathbf{i}$ & 0.8334298010 \\
11 & $0.2924975517-0.9562662716 \mathbf{i}$ & 0.3518577037 \\
12 & $0.8079717966-0.5892211604 \mathbf{i}$ & 0.2926150925 \\
\hline
\end{tabular}

where $\omega_{i}(\theta)$ is given by (6), and let

$$
f_{1}(z)=\frac{(z-1 / 4) \sin (z)}{8 \pi z}, \quad f_{2}(z)=\frac{(4-z) \sin (1 / z)}{8 \pi z} \quad \text { and } \quad f_{3}(z)=\frac{\sin (z)}{8 \pi z} .
$$

For these functions, we have used Maple 10 with 40 digits to compute the absolute error of the $n$-point Szegö quadrature formula, i.e.,

$$
e_{\tilde{\mu}}\left(f_{j}\right)=\left|I_{\tilde{\mu}}\left(f_{j}\right)-I_{n}\left(f_{j}\right)\right|, \quad j=1,2,3,
$$

for $n=8,16,24$ with $\tau_{n}=1$. The results are given in Tables $3[5$. 
TABLE 3 . The absolute error $e_{\tilde{\mu}}\left(f_{1}\right)$ for the $n$-point Szegö quadrature formula with respect to the rational modification of the weight functions $\omega_{i}(\theta)$.

\begin{tabular}{|c|ccc|}
\hline & $i=2$ & $i=3$ & $i=4$ \\
\hline$n=8$ & $4.381892940 \times 10^{-8}$ & $6.032565503 \times 10^{-7}$ & $7.738268455 \times 10^{-7}$ \\
$n=16$ & $4.417815525 \times 10^{-17}$ & $6.151507562 \times 10^{-16}$ & $7.903210143 \times 10^{-16}$ \\
$n=24$ & $1.010117754 \times 10^{-27}$ & $1.410431794 \times 10^{-26}$ & $1.812756380 \times 10^{-26}$ \\
\hline
\end{tabular}

TABle 4 . The absolute error $e_{\tilde{\mu}}\left(f_{2}\right)$ for the $n$-point Szegó quadrature formula with respect to the rational modification of the weight functions $\omega_{i}(\theta)$.

\begin{tabular}{|c|ccc|}
\hline & $i=2$ & $i=3$ & $i=4$ \\
\hline$n=8$ & $5.094348466 \times 10^{-5}$ & $9.843173970 \times 10^{-5}$ & $9.705465650 \times 10^{-5}$ \\
$n=16$ & $1.925426989 \times 10^{-13}$ & $3.815676087 \times 10^{-13}$ & $3.801621369 \times 10^{-13}$ \\
$n=24$ & $9.701673019 \times 10^{-24}$ & $1.932272042 \times 10^{-23}$ & $1.92904885 \times 10^{-23}$ \\
\hline
\end{tabular}

TABLE 5. The absolute error $e_{\tilde{\mu}}\left(f_{3}\right)$ for the $n$-point Szegö quadrature formula with respect to the rational modification of the weight functions $\omega_{i}(\theta)$.

\begin{tabular}{|c|ccc|}
\hline & $i=2$ & $i=3$ & $i=4$ \\
\hline$n=8$ & $1.750776412 \times 10^{-7}$ & $3.438809772 \times 10^{-7}$ & $3.376229665 \times 10^{-7}$ \\
$n=16$ & $1.766481103 \times 10^{-16}$ & $3.512396863 \times 10^{-16}$ & $3.491851390 \times 10^{-16}$ \\
$n=24$ & $4.039751881 \times 10^{-27}$ & $8.056536588 \times 10^{-27}$ & $8.033580897 \times 10^{-27}$ \\
\hline
\end{tabular}

\section{Appendix}

Theorem 7.1. There are no $\tau_{n} \in \mathbb{T}$ and $\gamma \in \mathbb{T} \backslash\left\{\nu_{i}\right\}$ so that $p_{1}^{(i) *}(\gamma)=0$.

Proof. Suppose there are $\tau_{n} \in \mathbb{T}$ and $\gamma \in \mathbb{T} \backslash\left\{\nu_{i}\right\}$ so that $\tau_{n} p_{1}^{(i) *}(\gamma)=0$. Clearly, it then holds that $p_{1}^{(i)}(\gamma)=0$ as well. Hence, from (27) we then deduce that

$$
\tau_{n}=-\frac{a_{n}^{(i)}\left(1-\overline{\alpha_{n}} \gamma\right)}{1-\overline{b_{n}^{(i)}} \gamma}=-\frac{\gamma-b_{n}(i)}{\overline{a_{n}^{(i)}}\left(\gamma-\alpha_{n}\right)}, \quad \gamma \in \mathbb{T} \backslash\left\{\nu_{i}\right\} .
$$

Therefore,

$$
\begin{aligned}
0 & =\gamma\left(\left|\gamma-b_{n}^{(i)}\right|^{2}-\left|a_{n}^{(i)}\right|^{2}\left|\gamma-\alpha_{n}\right|^{2}\right) \\
& =\left(\left|a_{n}^{(i)}\right|^{2} \overline{\alpha_{n}}-\overline{b_{n}^{(i)}}\right) \gamma^{2}+\left[1+\left|b_{n}^{(i)}\right|^{2}-\left|a_{n}^{(i)}\right|^{2}\left(1+\left|\alpha_{n}\right|^{2}\right)\right] \gamma+\left(\left|a_{n}^{(i)}\right|^{2} \alpha_{n}-b_{n}^{(i)}\right) \\
& =\left(\gamma-\nu_{i}\right)\left[\left(\left|a_{n}^{(i)}\right|^{2} \overline{\alpha_{n}}-\overline{b_{n}^{(i)}}\right) \gamma-\left(\left|a_{n}^{(i)}\right|^{2} \alpha_{n}-b_{n}^{(i)}\right)\right], \quad \gamma \in \mathbb{T} \backslash\left\{\nu_{i}\right\},
\end{aligned}
$$


where the last equality is due to the fact that the equality in (47) clearly holds for $\gamma=\nu_{i}$. Since we assumed $\gamma \neq \nu_{i}$, it follows that

$$
\gamma=\nu_{i} \frac{\left|a_{n}^{(i)}\right|^{2} \alpha_{n}-b_{n}^{(i)}}{\left|a_{n}^{(i)}\right|^{2} \overline{\alpha_{n}}-\overline{b_{n}^{(i)}}} .
$$

But from (10) we deduce that

$$
\left|a_{n}^{(i)}\right|^{2} \alpha_{n}-b_{n}^{(i)}=\frac{2 \Re\left\{\alpha_{n}\right\}-\nu_{i}\left[\left|1-\nu_{i} \overline{\alpha_{n}}\right|^{2} Q_{n}^{(i)}+1\right]}{\left|\left(1-\nu_{i} \overline{\alpha_{n}}\right) Q_{n}^{(i)}+1\right|^{2}}-\nu_{i} \in \mathbb{R},
$$

so we again find that $\gamma$ should equal $\nu_{i}$.

Theorem 7.2. If $n=1$ or $\alpha_{n}=0$, there are no $\tau_{n} \in \mathbb{T}$ and $\gamma \in \mathbb{T} \backslash\{ \pm 1\}$ so that $\tau_{n} p_{2}^{(2) *}(\gamma)=0$.

Proof. Similarly, as in the proof of Theorem 7.1. we find that there are $\tau_{n} \in \mathbb{T}$ and $\gamma \in \mathbb{T} \backslash\{ \pm 1\}$ so that $\tau_{n} p_{2}^{(2) *}(\gamma)=0$ iff

$$
\begin{aligned}
0 & =\gamma^{2}\left(\left|f_{n}+g_{n} \gamma+\gamma^{2}\right|^{2}-\left|d_{n}+e_{n} \gamma\right|^{2}\left|\gamma-\alpha_{n}\right|^{2}\right) \\
& =\left(\gamma^{2}-1\right)\left[\left(e_{n} \overline{d_{n}} \overline{\alpha_{n}}+\overline{f_{n}}\right) \gamma^{2}+\varpi_{n} \gamma-\left(\overline{e_{n}} d_{n} \alpha_{n}+f_{n}\right)\right], \quad \gamma \in \mathbb{T} \backslash\{ \pm 1\},
\end{aligned}
$$

where the last equality is due to the fact that the equality in (48) clearly holds for $\gamma= \pm 1$, and $\varpi_{n} \in \mathbb{R}$ is given by

$$
\begin{aligned}
\varpi_{n} & =g_{n}+f_{n} \overline{g_{n}}+\alpha_{n}\left(\left|d_{n}\right|^{2}+\left|e_{n}\right|^{2}\right)-d_{n} \overline{e_{n}}\left(1+\left|\alpha_{n}\right|^{2}\right) \\
& =\overline{g_{n}}+g_{n} \overline{f_{n}}+\overline{\alpha_{n}}\left(\left|d_{n}\right|^{2}+\left|e_{n}\right|^{2}\right)-e_{n} \overline{\bar{d}_{n}}\left(1+\left|\alpha_{n}\right|^{2}\right) .
\end{aligned}
$$

Since we assumed $\gamma \notin\{ \pm 1\}$, it should hold that

$$
\left(e_{n} \overline{d_{n}} \overline{\alpha_{n}}+\overline{f_{n}}\right) \gamma^{2}+\varpi_{n} \gamma-\left(\overline{e_{n}} d_{n} \alpha_{n}+f_{n}\right)=0 .
$$

Note that for the parameters $a^{+}, a^{-}, b^{+}$and $b^{-}$, given by (14), we have that

$$
\begin{array}{ll}
\overline{a^{+}}=\frac{1}{a^{+}\left|1-\alpha_{n}\right|^{2}}, & \overline{a^{-}}=\frac{1}{a^{-}\left|1+\alpha_{n}\right|^{2}}, \\
\overline{b^{+}}=b^{+}+\frac{2 i \Im\left\{\alpha_{n}\right\}}{\left|1-\alpha_{n}\right|^{2}}, & \overline{b^{-}}=b^{-}-\frac{2 i \Im\left\{\alpha_{n}\right\}}{\left|1+\alpha_{n}\right|^{2}} .
\end{array}
$$

With this observation in mind, we computed $\varpi_{n}$ with the aid of Maple 10 to find that $\varpi_{n}=0$, so it should hold that

$$
\gamma^{2}=\frac{\overline{e_{n}} d_{n} \alpha_{n}+f_{n}}{e_{n} \overline{d_{n}} \overline{\alpha_{n}}+\overline{f_{n}}}
$$

For the special case in which $\alpha_{n}=0$, it follows from (18) that $f_{n}=\frac{-2}{\pi\left|h_{n}\right|^{2}} \in \mathbb{R}$, so we again find that $\gamma$ should equal \pm 1 . For $n=1$, on the other hand, we have that

$$
a^{+}=\frac{1}{1-\overline{\alpha_{1}}}, \quad a^{-}=\frac{1}{1+\overline{\alpha_{1}}}, \quad b^{+}=2+a^{+} \quad \text { and } \quad b^{-}=2+a^{-} .
$$

We then computed $\gamma^{2}$, given by (49) with $n=1$, to find that $\gamma^{2}=1$. At this moment of writing, however, we could not verify whether $\gamma^{2}$, given by (49), equals one for the more general case of $\alpha_{n} \neq 0$ and $n>1$. 


\section{REFERENCES}

1. A. Bultheel, L. Daruis, and P. González-Vera, A connection between quadrature formulas on the unit circle and the interval $[-1,1]$, J. Comput. Appl. Math. 132 (2000), no. 1, 1-14. MR.1834799 (2002c:65042)

2. put. Appl. Math. 179 (2005), no. 1-2, 97-119. MR2134362 (2005m:41061)

3. A. Bultheel, P. González-Vera, E. Hendriksen, and O. Njåstad, Orthogonal rational functions and quadrature on the unit circle, Numer. Algorithms 3 (1992), 105-116. MR1199359 (94a:42022)

4. , Quadrature formulas on the unit circle based on rational functions, J. Comput. Appl. Math. 50 (1994), 159-170. MR:1284259 (95h:41053)

5. - Orthogonal rational functions, Cambridge Monographs on Applied and Computational Mathematics, vol. 5, Cambridge University Press, 1999. MR.1676258 (2000c:33001)

6. — Quadrature and orthogonal rational functions, J. Comput. Appl. Math. 127 (2001), no. 1-2, 67-91, Invited paper. MR1808569 (2001m:65036)

7. _ Rational quadrature formulas on the unit circle with prescribed nodes and maximal domain of validity, (2007), Submitted.

8. M.J. Cantero, R. Cruz-Barroso, and P. González-Vera, A matrix approach to the computation of quadrature formulas on the interval, Appl. Numer. Math. 58 (2008), no. 3, 296-318. MR.2392689

9. R. Cruz-Barroso, L. Daruis, P. González-Vera, and O. Njåstad, Sequences of orthogonal Laurent polynomials, bi-orthogonality and quadrature formulas, J. Comput. Appl. Math. 200 (2006), 424-440. MR.2333724 (2008f:65042)

10. L. Daruis and P. González-Vera, Szegö polynomials and quadrature formulas on the unit circle, Appl. Numer. Math. 36 (2000), no. 1, 79-112. MR1808125 (2001m:65037)

11. L. Daruis, P. González-Vera, and O. Njåstad, Szegő quadrature formulas for certain Jacobitype weight functions, Math. Comp. 71 (2002), no. 238, 683-701. MR1885621 (2002k:41043)

12. L. Daruis, P. González-Vera, and M. Jiménez Paiz, Quadrature formulas associated with rational modifications of the Chebyshev weight functions, Comput. Math. Appl. 51 (2006), 419-430. MR2207429 (2006k:65057)

13. P.J. Davis, Interpolation and approximation, Dover Publications, New York, 1975. MR 0380189 (52:1089)

14. K. Deckers and A. Bultheel, Orthogonal rational functions and rational modifications of a measure on the unit circle, J. Approx. Theory (2008), Accepted.

15. K. Deckers, J. Van Deun, and A. Bultheel, Computing rational Gauss-Chebyshev quadrature formulas with complex poles, Proceedings of the Fifth International Conference on Engineering Computational Technology (Kippen, Stirlingshire, United Kingdom) (B.H.V. Topping, G. Montero, and R. Montenegro, eds.), Civil-Comp Press, 2006, Paper 30.

16. An extended relation between orthogonal rational functions on the unit circle and the interval $[-1,1]$, J. Math. Anal. Appl. 334 (2007), no. 2, 1260-1275. MR2338662|(2008h:42044)

17. _ Computing rational Gauss-Chebyshev quadrature formulas with complex poles: the algorithm, Advances in Engineering Software (2008), Accepted.

18. _ Rational Gauss-Chebyshev quadrature formulas for complex poles outside [-1,1], Math. Comp. 77 (2008), no. 262, 967-983. MR2373187

19. W. Gautschi, A survey of Gauss-Christoffel quadrature formulae, E.B. Christoffel. The influence of his work on mathematical and physical sciences (Basel) (P.L. Butzer and F. Fehér, eds.), Birkhäuser Verlag, 1981, pp. 72-147. MR661060 (83g:41031)

20. W. Gautschi, L. Gori, and M.L. Lo Cascio, Quadrature rules for rational functions, Numer. Math. 86 (2000), no. 4, 617-633. MR1794345 (2002a:41030)

21. Ya. Geronimus, Polynomials orthogonal on a circle and interval, International Series of Monographs in Pure and Applied Mathematics, Pergamon Press, Oxford, 1960. MR0133642 (24:A3468)

22. W.B. Gragg, Positive definite Toeplitz matrices, the Arnoldi process for isometric operators, and Gaussian quadrature on the unit circle, J. Comput. Appl. Math. 46 (1993), 183-198. MR $1222480(94 \mathrm{e}: 65046)$

23. U. Grenander and G. Szegő, Toeplitz forms and their applications, Chelsea Publishing Company, New-York, 1958. MR.0094840 (20:1349) 
24. C. Jagels and L. Reichel, Szegö-Lobatto quadrature rules, J. Comput. Appl. Math. 200 (2007), no. 1, 116-126. MR2276819 (2008c:65074)

25. W.B. Jones, O. Njåstad, and W.J. Thron, Moment theory, orthogonal polynomials, quadrature and continued fractions associated with the unit circle, Bull. London Math. Soc. 21 (1989), 113-152. MR976057 (90e:42027)

26. B. Simon, Orthogonal polynomials on the unit circle. Part 1: Classical theory, Colloquium Publications, vol. 54, AMS, 2005. MR2105088(2006a:42002a)

27. G. Szegö, On bi-orthogonal systems of trigonometric polynomials, Magyar Tud. Alcad. Kutato Int. Közl. 8 (1963), 255-273. MR0166541 (29:3815)

28. _ Orthogonal polynomials, 4th ed., Amer. Math. Soc. Colloq. Publ., vol. 33, Amer. Math. Soc., Providence, Rhode Island, 1975.

29. W. Van Assche and I. Vanherwegen, Quadrature formulas based on rational interpolation, Math. Comp. 16 (1993), 765-783. MR1195424 (94a:65014)

30. J. Van Deun, A. Bultheel, and P. González-Vera, On computing rational Gauss-Chebyshev quadrature formulas, Math. Comp. 75 (2007), no. 253, 307-326. MR2176401(2006e:41060)

31. J. Van Deun, K. Deckers, A. Bultheel, and J.A.C. Weideman, Algorithm 882: Near best fixed pole rational interpolation with applications in spectral methods, ACM Trans. Math. Software 32 (2008), no. 2, article no. 14, pp. 1-21.

32. P. Van gucht and A. Bultheel, A relation between orthogonal rational functions on the unit circle and the interval $[-1,1]$, Comm. Anal. Th. Continued Fractions 8 (2000), 170-182. MR 1789681 (2001h:42037)

33. H. Waadeland, A Szegö quadrature formula for the Poisson integral, Computational and Applied Mathematics I (C. Brezinski and U. Kulish, eds.), Elsevier, 1992, pp. 479-486. MR $1203369(93 \mathrm{k}: 65022)$

Department of Computer Science, K.U.Leuven, Celestijnenlaan 200 A, B-3001 LeuVEN, Belgium.

E-mail address: Adhemar.Bultheel@cs.kuleuven.be

Department of Computer Science, K.U.Leuven, Celestijnenlaan 200 A, B-3001 LeuVen, Belgium.

Current address: Department of Mathematical Analysis, La Laguna University, 38271 La Laguna, Tenerife, Canary Islands, Spain

E-mail address: szegoquadrature@hotmail.com

Department of Computer Science, K.U.Leuven, Celestijnenlaan 200 A, B-3001 LeuVEN, Belgium.

E-mail address: Karl.Deckers@cs.kuleuven.be

Department of Mathematical Analysis, La Laguna University, 38271 La Laguna, Tenerife, Canary Islands, Spain

E-mail address: pglez@ull.es 